This document is the Accepted Manuscript version of a Published Work that appeared in final form in The Journal of Physical Chemistry C, copyright $@$ American Chemical Society after peer review and technical editing by the publisher. To access the final edited and published work see https://pubs.acs.org/articlesonrequest/AOR-q5bvXhfPFUXrki4QWjuj .

\title{
Understanding the Properties of Tailor-Made Self-Assembled Monolayers with Embedded Dipole Moments for Interface Engineering
}

Michael Gärtner, ${ }^{\dagger, \#}$ Eric Sauter, ${ }^{\ddagger}, \#$ Giulia Nascimbeni, ${ }^{\|, \# ~ A n d r e a s ~ P e t r i t z, ~}{ }^{\S}$ Adrian Wiesner, ${ }^{\dagger}$

Martin Kind, ${ }^{\dagger}$ Tarek Abu-Husein,${ }^{\dagger}$ Michael Bolte, ${ }^{\dagger}$ Barbara Stadlober, ${ }^{\S}$ Egbert Zojer,,${ }^{\Perp}$ Andreas Terfort, ${ }^{\dagger}, *$ and Michael Zharnikov ${ }^{\ddagger} * *$

'Institut für Anorganische und Analytische Chemie, Johann Wolfgang Goethe Universität Frankfurt, Max-von-Laue-Straße 7, D-60438 Frankfurt am Main, Germany

${ }^{*}$ Angewandte Physikalische Chemie, Universität Heidelberg, Im Neuenheimer Feld 253, D-69120 Heidelberg, Germany

"Institute of Solid State Physics, Graz University of Technology, Petersgasse 16, 8010 Graz, Austria

$\S_{\text {MATERIALS-Institute for Surface Technologies and Photonics, Joanneum Research }}$ Forschungsgesellschaft, Franz-Pichlerstrasse 30, 8160 Weiz, Austria.

\footnotetext{
\# these authors have provided equivalent contributions
} 


\begin{abstract}
Self-assembled monolayers (SAMs) are frequently used for interfacial dipole engineering in organic electronics (OE) and photovoltaics. This is mostly done by the attachment of dipolar tail groups onto the molecular backbone of the SAM precursors. The alternative concept of embedded dipoles involves the incorporation of polar group(s) into the backbone. This allows to decouple the tuning of the electrostatic properties of the SAM from the chemical identity of the SAM-ambient interface. Here we present design and synthesis of particularly promising SAM precursors utilizing this concept. These precursors feature the thiol docking group and a short heteroaromatic backbone, consisting of a non-polar phenyl ring and a polar pyrimidine group, embedded in two opposite orientations. Packing density, molecular orientation, structure and wetting properties of the SAMs on Au substrates are found to be nearly independent of their chemical structure, as shown by a variety of complementary experimental techniques. A further important property of the studied SAMs is their good electrical conductivity, enabling their application as electrode modifiers for low contact resistances in organic electronics devices. Of particular interest are also the electronic properties of the SAMs, which were monitored by Kelvin probe and high-resolution x-ray photoelectron spectroscopy measurements. To obtain a fundamental understanding of these properties at an atomistic level, the experiments are combined with state-of-the-art band structure calculations. These not only confirm the structural properties of the films but also explain, how the $\mathrm{C} 1$ s core-level binding energies of the various atoms are controlled by their chemical environments in conjunction with the local distribution of the electrostatic potential within the monolayer.
\end{abstract}




\section{Introduction}

Interfacial engineering in organic electronics is an important subject of current research. ${ }^{1-3}$ The most essential aspects within this topic are a) the optimization of the charge carrier injection barrier by minimizing the energetic gap between the electrode Fermi level and the transport levels of the organic semiconductor (OSC) and b) the surface-mediated growth of the active layer at the electrodes and in the channel. Both aspects can be addressed by modifying the electrodes or gate dielectric with self-assembled monolayers (SAMs), ${ }^{4-7}$ which are 2D polycrystalline films of semirigid molecules that are chemically bound to a substrate by suitable docking groups. ${ }^{8,9}$

A particularly important advantage of using SAMs is that they allow the optimization of charge carrier injection barriers at the electrode/OSC interface via the adjustment of the electrode work function (WF). Typically, the WF of an electrode increases when perfluorinated molecules are chemisorbed, while it decreases for the corresponding non-fluorinated, H-terminated derivatives. ${ }^{10,11}$ An additional tuning of the WF can then be achieved by attachment of polar tail groups like $-\mathrm{CN},-\mathrm{F},-\mathrm{CF}_{3},-\mathrm{NH}_{2}$, or $-\mathrm{NO}_{2}$ to the $\mathrm{SAM}$ backbone, which is probably the most frequently used approach in this regard. ${ }^{11-15}$ This strategy, however, has significant drawbacks, such as (i) a strong influence of the nature of the polar tail groups on OSC growth (vide supra) and (ii) the risk of a possible modification of the (chemical) structure of the tailgroup substituents as a consequence of the interaction with the eventually deposited OSC. These problems can be avoided by embedding the polar groups into the backbone of the SAM constituents. This permits an independent adjustment of the dipole moment and the interfacial chemistry at the SAM/OSC interface and prevents direct contact between the dipolar groups and the OSC. First examples of such SAMs comprised aliphatic backbones with embedded polar ester groups at varying orientations ${ }^{16,17}$ and the embedded dipole concept has recently also been used to explain the electronic properties of partially fluorinated aliphatic SAMs ${ }^{18}$ As an alternative, pyrimidine groups have been embedded into an aromatic backbone. ${ }^{19}$ Both for aliphatic and aromatic SAMs, the inclusion of the embedded dipoles results in only minor changes of the resulting monolayer structure as compared to the parent, non-substituted films. ${ }^{16,17,19}$ The SAMs not only allow increasing or decreasing the substrate work function (for dipole-down and dipole-up orientations, respectively), but enable also a continuous tuning of the WF between the ultimate values of the single-component SAMs by combining up- and down-constituents in mixed monolayers, ${ }^{7,20-22}$ in analogy to what has been observed also for mixtures of fluorinated and non-fluorinated alkanethiolate SAMs. ${ }^{23,24}$ 
The initial design of the prototypical SAMs with embedded dipoles, however, disregarded an important aspect, namely the electrical resistance caused by the organic monolayer, which reduces the efficiency of charge carrier injection at electrode-SAM-OSC interfaces. This is, on the one hand, a consequence of the transport properties of aliphatic SAMs, which are inferior to their aromatic counterparts. ${ }^{25-27}$ On the other hand, the prototypical (first generation) aromatic SAMs with embedded dipoles tested in recent years relied on comparably long backbones consisting of three rings and a methylene linker connecting the backbone to the thiolate docking group (see Figure S1 in the Supporting Information). ${ }^{19}$ The latter has been introduced primarily to improve molecular packing and lateral order in the SAMs. ${ }^{28-30}$ Significantly, both long backbones and methylene linkers are detrimental to charge transport through the SAMs, as the conductance of molecular monolayers typically decreases exponentially with film thickness, ${ }^{25-27,31}$ while the introduction of the methylene linker electronically decouples the metal substrate from the conjugated segment. ${ }^{32-34}$

Bearing the above considerations in mind, we designed a set of molecules (second generation) that exploits the concept of embedded dipoles in a fashion much more suitable for applications in organic electronic devices (Figure 1). As polar element (yellow in Figure 1), a pyrimidine ring ("Pm") with a dipole moment of 2.3 Debye $^{35}$ was used. It was built into a biaryl system in two different orientations. To make sure that in all cases the same tail group (green in Figure 1) is exposed at the SAM/OSC interface, a phenyl ring ("P") was employed as second aryl element. As anchoring group, the thiol group (orange in Figure 1) is directly attached to the aromatic system in a way that no other heteroatoms are close to it, as it has been shown that the presence of heteroatoms, such as $\mathrm{N}$ or $\mathrm{O}$, often hampers the formation of high quality SAMs. ${ }^{36-}$ ${ }^{38}$ As reference, 4-biphenyl thiol (PP), with a non-polar backbone, was used. In some sense, PP can be considered as "parent" molecule for PmP-up and PPm-down, where one of the phenyl rings is substituted by pyrimidine.

We have shown recently that SAMs consisting of PmP-up and PPm-down molecules can reduce or increase, respectively, contact resistances in p- and n-type transistors comprising established organic semiconductors (pentacene and $\mathrm{C}_{60}$ ). ${ }^{39}$ In the same study we also extensively characterized the properties of the pentacene layers grown on top of the SAM-modified electrodes, showing that there is only little difference in their structure and morphology for different dipole orientations. In the present work, we focus on the characteristics of the SAMs themselves, describing the synthesis of the precursors and studying their structural properties by ellipsometry, infrared reflection-absorption spectroscopy (IRRAS), scanning-tunneling 
microscopy (STM), near edge x-ray absorption fine structure (NEXAFS) spectroscopy, and contact angle goniometry. Moreover, x-ray photoelectron spectroscopy (XPS) reveals the integrity of the SAMs. In conjunction with dispersion-corrected density-functional theory (DFT) based simulations, it also provides insight into the electronic structure within the SAMs, correlating core-level binding energies with local shifts in the local electrostatic potential. The latter also gives rise to SAM-induced work function changes, which are quantified by the Kelvin Probe (KP) technique. Finally, we briefly review the charge-transport characteristics through the SAMs and their application as electrode modifiers in p- and n-type transistors.

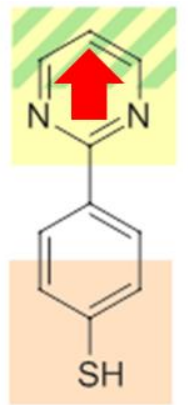

PmP-up

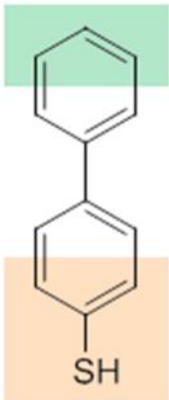

PP

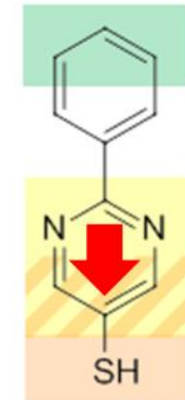

PPm-down

Figure 1: SAM-forming molecules used in this project along with their acronyms $(\mathbf{P}=$ phenyl, $\mathbf{P m}=$ pyrimidine, $\mathbf{u p} / \mathbf{d o w n}=$ direction of dipole moment (red arrows) with respect to the anchoring group). The structure of this series was optimized for the application of the corresponding SAMs in organic electronics and photovoltaics. Chemically similar parts of the molecular building blocks are marked by different colors (see text for details), with mixed colors reflecting the possible influence of the adjacent nitrogen atoms at the respective interface upon monomolecular assembly. Note that common acronyms for PP are also BPT (biphenylthiol) or BP0 and that, consequently, BP0-up and BP0-down has been used by us before for PmP-up and PPm-down. ${ }^{39} \mathrm{We}$, however, feel that the acronyms used in this work better reflect the molecular identity of the system and are also more easily adapted to more complex molecular structures (containing, e.g., multiple pyrimidine units).

\section{Experimental}

Chemicals and Synthesis Procedure: Solvents and chemicals necessary for the synthesis of the SAM precursors (Figure 1) were purchased from different vendors (see the Supporting 
Information) and used as received. PP was synthesized according to a literature procedure. ${ }^{40}$ To synthesize PPm-down, a triisopropylsilylsulfide group was introduced to 5-bromo-2-phenylpyrimidine by a cross coupling reaction with triisopropylsilythiol, $\mathrm{Pd}(\mathrm{dppf}) \mathrm{Cl}_{2}$ as catalyst and LiHMDS as base. By reaction with $\mathrm{HCl}$ in $\mathrm{MeOH}$, the TIPS-group was cleaved to release the free thiol. For the synthesis of PmP-up, 4-bromophenyl(triisopropylsilyl)sulfide was first converted to the corresponding Grignard reagent and coupled with 2-chloropyrimidine catalyzed by $\mathrm{Pd}(\mathrm{dppf}) \mathrm{Cl}_{2}$ followed by deprotection with $\mathrm{HCl}$ in $\mathrm{MeOH}$. A detailed description of the synthesis procedures and the characterization data for the intermediates and final products are given in the Supporting Information.

X-ray Diffraction Analysis: The bulk structures of the single crystals of PPm-down and PmPup were determined by X-ray diffraction. The data were collected on a STOE IPDS II twocircle diffractometer with a Genix Microfocus tube with mirror optics using Mo Ka radiation $(\lambda=0.71073 \AA)$. The data were scaled using the frame-scaling procedure in the $X$-AREA program system. ${ }^{41}$ The structures were solved by direct methods using the program SHELXS ${ }^{42}$ and refined against $F^{2}$ with full-matrix least-squares techniques again employing SHELXL. ${ }^{42}$ The CCDC deposition numbers are CCDC-1865408 (PmP-up) and CCDC-1865409 (PPmdown). PmP-up: The $\mathrm{H}$ atom bonded to $\mathrm{S}$ is disordered over two equally occupied positions. Nevertheless, it was freely refined. PPm-down: The coordinates of the $\mathrm{H}$-atom bonded to $\mathrm{S}$ were refined. Its $U$-value was set to $1.5 U_{\mathrm{eq}}(\mathrm{S})$.

SAM Preparation: The PPm-down, PmP-up, and PP SAMs were prepared on gold substrates. The substrates were purchased from Georg Albert PVD- Beschichtungen (Silz, Germany). They were prepared by thermal evaporation of $30 \mathrm{~nm}$ of gold (99.99\% purity) onto polished single-crystal silicon (100) wafers (Silicon Sense) that had been precoated with a $9 \mathrm{~nm}$ titanium adhesion layer. The films were polycrystalline, exposing mostly (111) orientated surfaces of individual crystallites. The RMS value for these substrates was estimated as $0.8 \mathrm{~nm}\left(5 \times 5 \mu \mathrm{m}^{2}\right.$ scan area); in our experience, this value does not change noticeably upon the SAM formation.

Substrates for scanning tunneling microscopy (STM) measurements were purchased from Phasis (Geneva) or prepared by e-beam evaporation of gold $(200 \mathrm{~nm})$ onto high quality mica at $400{ }^{\circ} \mathrm{C}$ in ultra-high vacuum (UHV). Before evaporation the mica substrates were annealed for $3 \mathrm{~h}$ at $400{ }^{\circ} \mathrm{C}$. First $150 \mathrm{~nm}$ of gold were deposited at a high evaporation rate (30-60 $\left.\mathrm{\AA} / \mathrm{s}\right)$ followed by $50 \mathrm{~nm}$ at a lower rate $(0.5 \AA / \mathrm{s})$. A shutter was used to protect the substrate while adjusting the evaporation rate. After evaporation the substrates were annealed for $1 \mathrm{~h}$ at $400{ }^{\circ} \mathrm{C}$.

The SAMs were formed by immersion of the substrates into solutions of the SAM precursors 
in either tetrahydrofuran (THF; Sigma-Aldrich) or ethanol (Sigma-Aldrich) for $24 \mathrm{~h}$ (also for a longer time, in the case of the STM experiments; see section 3.5) under nitrogen and at room temperature. After immersion, the samples were carefully rinsed with pure solvent and dried under a flow of $\mathrm{N}_{2}$. The SAMs prepared from both solvents did not show any significant differences, with only one exception (see section 3.4); therefore, mostly the data for the THF preparation are shown). Note that THF provides a better solubility of the thiols compared to ethanol and also, in the given case, a better reproducibility of the monolayers. However, in our previous study, dealing with the device applications of the PPm-down and PmP-up SAMs, we used the ethanol preparation, because of specific technical reasons. ${ }^{39}$

In addition, reference SAMs of hexadecanethiolate (HDT) and perdeuterated dodecanethiolate on the same $\mathrm{Au}(111)$ substrates were prepared according to the literature procedures. ${ }^{43,44} \mathrm{HDT}$ was purchased from Sigma-Aldrich; the perdeuterated dodecanethiol was synthesized from the respective bromoalkane using standard procedures.

Characterization: General Comments: The SAMs were experimentally characterized by ellipsometry, contact angle goniometry, STM, IRRAS, synchrotron-based XPS, NEXAFS spectroscopy, KP measurements, and electric conductance measurements. In all cases, the characterization was performed at room temperature. XPS and NEXAFS spectroscopy experiments were conducted under UHV conditions, with special care taken to minimize potential modification of the SAMs induced by the primary X-rays. ${ }^{45-47}$ The SAMs were also characterized computationally by means of quantum mechanical simulations to analyze the experimental data and to support their interpretation. Note that a limited characterization of the PPm-down and PmP-up SAMs prepared from EtOH on "technical" gold substrates also used for device fabrication (for details see ref 39) has already been performed within the previous device study (contact angle goniometry, work function, and electric conductance). ${ }^{39}$

Ellipsometry: Ellipsometry measurements on the SAMs were carried out with a Sentech SE 400 ellipsometer equipped with a He/Ne laser (wavelength $632.8 \mathrm{~nm}$, beam diameter 1-2 mm). The angle of incidence was $70^{\circ}$ with respect to the sample surface normal. The complex refractive indices of the substrates, necessary for the data evaluation, were measured separately after a hydrogen plasma treatment for $2 \mathrm{~min} .{ }^{48}$ For the refractive indices of the monolayers, the extinction coefficients were assumed to be zero, while the real part was assumed to be 1.55 , a value that, according to our experience, is well applicable in the case of mainly aromatic molecules.

Contact Angle Goniometry: Advancing and receding contact angles of millipore water were 
measured on freshly prepared samples with a custom-made, computer-controlled goniometer. The measurements were performed under ambient conditions with the needle tip in contact with the drop. At least three measurements at different locations on each sample were made. The averaged values are reported. Deviations from the average were less than $\pm 2^{\circ}$.

IRRAS: All IR spectra were obtained using a Thermo Nicolet 6700 Fourier transform IR spectrometer with a narrow-band mercury cadmium telluride semiconductor detector at a resolution of $4 \mathrm{~cm}^{-1}$. The optical path was purged with dried and $\mathrm{CO}_{2}$-free air during the measurement. Neat substances were measured using a single-reflection diamond attenuated total reflection unit and the infrared reflection absorption spectra of the SAMs were recorded at an angle of $80^{\circ}$ relative to the sample surface normal with $p$-polarized radiation against a reference SAM of perdeuterated dodecanethiolate on $\mathrm{Au}$.

IR spectra of isolated molecules were calculated using density functional theory (Gaussian 03 program package ${ }^{49}$ with the BP86 functional ${ }^{50,51}$ and the SVP basis set ${ }^{52}$ ), helping to assign the vibrational modes and to identify the directions of their transition dipole moments (TDMs). Spectra were not scaled.

STM: A Bruker Multimode 8 Nanoscope with a MultiMode V SPM Control Station was used for STM experiments. A low-current STM Converter (Model MMSTMLCE) allowed carrying out measurements in the pA regime. Therefore, a usual measurement current was 5-50 pA at a sample bias of about $200 \mathrm{mV}$. The scanner had a maximum range of $1.4 \mu \mathrm{m} \times 1.4 \mu \mathrm{m}$. Probes were manufactured by cutting Pt-Ir (80:20) wires with a diameter of $0.25 \mathrm{~mm}$. The tip quality was assessed by measuring highly oriented pyrolytic graphite (HOPG). Only monolayers deposited onto gold on mica substrates (vide infra) were measured.

XPS: XPS measurements were performed at the HE-SGM beamline (bending magnet) of the synchrotron storage ring BESSY II in Berlin, Germany, using a custom-made experimental station. ${ }^{53}$ Primary photon energies (PE) of $350 \mathrm{eV}$ and $580 \mathrm{eV}$ were used. The spectra acquisition was carried out in normal emission geometry with a Scienta R3000 electron energy analyzer. The energy resolution was $\sim 0.3 \mathrm{eV}$ and $\sim 0.6 \mathrm{eV}$ at PEs of $350 \mathrm{eV}$ and $580 \mathrm{eV}$, respectively.

The binding energy (BE) scale of the spectra was referenced to the $A u 4 f_{7 / 2}$ emission at 84.0 $\mathrm{eV} .{ }^{54}$ When necessary, the spectra were fitted by symmetric Voigt functions and either a linear or Shirley-type background. To fit the $S 2 p_{3 / 2,1 / 2}$ doublets, we used two peaks with the same full 
width at half-maximum (fwhm), a standard ${ }^{54}$ spin-orbit splitting of $\sim 1.2 \mathrm{eV}$ (verified by a fit), and a branching ratio of $2\left(\mathrm{~S} 2 \mathrm{p}_{3 / 2} / \mathrm{S} 2 \mathrm{p}_{1 / 2}\right)$.

The effective thicknesses of the SAMs and their packing densities were calculated using standard procedures, ${ }^{43,55}$ based on the $\mathrm{C} 1 \mathrm{~s} / \mathrm{Au} 4 \mathrm{f}$ and $\mathrm{S} 2 \mathrm{p} / \mathrm{Au} 4 \mathrm{f}$ intensity ratios, respectively. For the thickness evaluation, a standard expression for the attenuation of the photoemission signal was assumed ${ }^{56}$ and the literature values for attenuation lengths, relying on the measurements of alkanethiolate SAMs, were used, viz. 11.5 and $15.75 \AA$ for kinetic energies of 295 and $490 \mathrm{eV}$, respectively. ${ }^{57}$ The spectrometer-specific coefficients were determined by using the PP SAM as a direct reference, relying on the well-known thickness of this monolayer $(1.09 \pm 0.02 \mathrm{~nm})^{44}$. This SAM also served as a reference for the evaluation of packing densities, relying on the packing density $\left(4.63 \times 10^{14}\right.$ molecules $\left./ \mathrm{cm}^{2}\right)$ corresponding to the $\sqrt{3} \times \sqrt{3}$ molecular lattice, which is the dominant structural phase of the PP SAMs formed after long immersion time. ${ }^{58,59}$ This value was additionally verified by referencing the PP SAM to the even better defined HDT monolayer on $\mathrm{Au}(111)$, also having an overall packing density of $4.63 \times 10^{14}$ molecules $/ \mathrm{cm}^{2}{ }^{60}$ The packing density of the PP SAM was found to be nearly identical to that of HDT, being only slightly $(\sim 4 \%)$ lower, which is presumably related to the specific morphology of the PP monolayer (small domains and a certain polymorphism). ${ }^{59}$

NEXAFS Spectroscopy: The NEXAFS spectra were collected at the same beamline as the XPS data. They were measured at the carbon and nitrogen K-edges in the partial electron yield (PEY) mode with retarding voltages of $-150 \mathrm{~V}$ and $-300 \mathrm{~V}$, respectively. Linearly polarized synchrotron light with a polarization factor of $\sim 89 \%$ was used as the primary X-ray source. The incidence angle of the X-rays was varied between the normal $\left(90^{\circ}\right)$ and grazing $\left(20^{\circ}\right)$ incidence geometry to monitor the linear dichroism reflecting the molecular orientation in the SAMs. ${ }^{61}$ The energy resolution was $\sim 0.3 \mathrm{eV}$ at the $\mathrm{C}$ K-edge and $\sim 0.45 \mathrm{eV}$ at the $\mathrm{N}$ K-edge. The PE scale was referenced to the pronounced $\pi^{*}$ resonance of HOPG at $285.38 \mathrm{eV}{ }^{62}$ The spectra were corrected for the PE dependence of the incident photon flux and reduced to the standard form with zero intensity in the pre-edge region and the unity jump in the far post-edge region.

KP Measurements: Work function measurements were carried out using a UHV Kelvin Probe 2001 system (KP technology Ltd., UK). The pressure in the UHV chamber was $\sim 10^{-9} \mathrm{mbar}$. As reference, we used a HDT SAM with the work function value set to $4.3 \mathrm{eV}$ according to literature. ${ }^{17}$ The latter value was additionally verified by its referencing to the work function of freshly sputtered gold set to $5.2 \mathrm{eV}^{63}$ 
Electrical Conductance Measurements: The measurements were performed with a custommade two-terminal tunneling junction setup, based on the Keithley 2635A source meter. ${ }^{64}$ The gold substrate and a sharp tip of eutectic GaIn (EGaIn) ${ }^{65}$ served as bottom and top electrodes. ${ }^{65}$ Tunneling junctions were formed by contacting grounded SAM/Au samples with the EGaIn tips and applying a potential. The voltage was varied between -0.6 and $+0.6 \mathrm{~V}$ in steps of 0.05 $\mathrm{V}$. At least $10 \mathrm{I}-\mathrm{V}$ curves measured at several different places were recorded for each sample; average values were calculated.

Quantum Mechanical Simulations: The computational study was performed using the FHIaims code ${ }^{66}$ employing the PBE functional. ${ }^{67}$ To account for long-range van der Waals interactions, we employed the surface version ${ }^{68}$ of the Tkatchenko-Scheffler dispersion corrections ${ }^{69}$. The system was modeled using periodic boundary conditions and the repeated slab approach. The metallic substrate was described with five Au layers, holding the three bottom ones fixed during all calculations and turning off the dispersion corrections between the $\mathrm{Au}$ atoms. Two molecules arranged in a herringbone fashion were put in a $(3 \times \sqrt{3})$ rect unit cell, whose dimensions in the $x$ and $y$ directions were defined according to the calculated Au lattice constant and held fixed in all calculations. The systems were optimized using the FHI-aims default "tight" setting and a $9 \times 5 \times 1 \Gamma$ centered k-points grid. The total energy criterion for the self-consistency cycle was set to $10^{-6} \mathrm{eV}$ and geometry optimizations were continued until the maximum residual force component per atom was below $0.01 \mathrm{eV} / \AA$.

The C 1s XP spectra were simulated within the initial state approach, to avoid artifacts arising from combining periodic boundary conditions and explicit excitations in each unit cell. ${ }^{70}$ The spectra were modeled following the procedure described in ref 70 using an image potential model to account for the screening due to the highly polarizable metal substrate and weighting the contribution of every atom to account for the finite escape depth of the photoelectrons.

Further information regarding the initial geometry, the numerical settings and the simulation of the XP spectra can be found in the Supporting Information.

\section{Results and Discussion}

\subsection{Synthesis}

As mentioned in section 2, the new pyrimidine-containing molecules were obtained via palladium-catalyzed cross-coupling reactions from literature-known starting materials (Scheme 
1). ${ }^{71,72}$ The use of the triisopropylsilyl (TIPS) moiety as protective group for the sulfur atoms was a key to this procedure, as this group is compatible with the Pd chemistry. ${ }^{72,73}$

It is worth mentioning that the TIPS derivatives of thiophenols are, in contrast to aliphatic TIPSprotected thiols, not very stable against the typical conditions of column chromatography or gradient sublimation. Therefore, the intermediates $\mathbf{2}$ and $\mathbf{4}$ were not isolated, but the crude products were directly deprotected following a literature known procedure. ${ }^{19}$ The resulting thiols (PPm-down and PmP-up) are very prone to oxidation during the typical work-up procedures, resulting in only moderate yields.
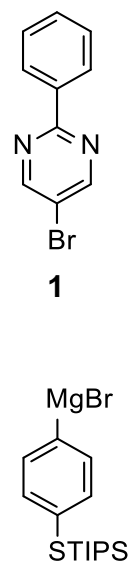

3

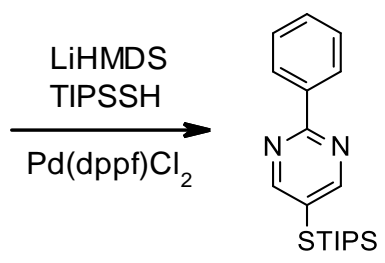

2

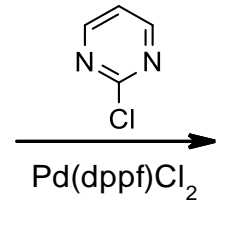

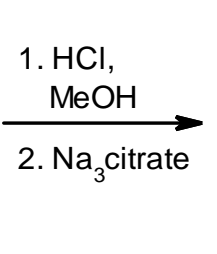

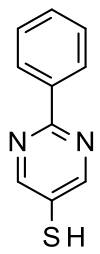

PPm-down

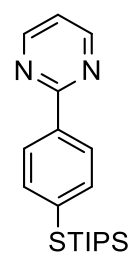

4

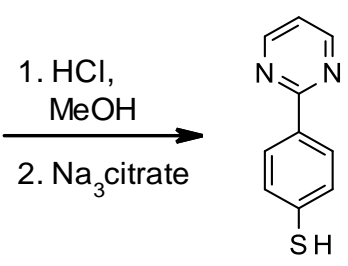

PmP-up

Scheme 1: Synthesis of dipolar SAM precursors 2-phenylpyrimidine-5-thiol (PPm-down) and 2-(4-mercaptophenyl)pyrimidine (PmP-up).

\subsection{X-ray diffraction analysis}

In spite of the proneness of PPm-down and PmP-up to oxidation, it was nevertheless possible to obtain single crystals of both compounds and to determine the respective bulk structure by X-ray diffraction (see the Supporting Information, in particular Figures S9 and S11). Both structures have two features in common, viz. an antiparallel arrangement of the molecules compensating the molecular dipole moments and a close-to-coplanar arrangement of the aromatic rings within individual molecules. While for PmP-up the torsion is less than $1^{\circ}$, for PPm-down, an angle of about $17^{\circ}$ could be determined. This is presumably due to sterical interactions and the specific packing motif mentioned above. The frequently observed coplanarity of 2-phenylpyrimidines ${ }^{74}$ provides a good electronic coupling between the $\pi$ systems of individual rings, which is expected to be advantageous for the electric conductivity in the respective SAMs, provided that the bulk arrangement is mimicked in the monolayers (apart from the antiparallel arrangement not observed in the monolayers; see below). 
In spite of the above similarities, there are certain differences in the packing of the molecules (see the Supporting Information), presumably because in the case of PPm-down an interaction between the $\pi$-system of the phenyl ring and the thiol proton of a neighboring molecule occurs, resulting in the formation of distinguished pairs. For PmP-up, this interaction would need to occur with the pyrimidine ring, which has a lower electron density.

Note that although the X-ray diffraction analysis of the single crystals provides important reference data for the SAMs, such as a close-to-coplanar orientation of the aromatic rings within individual molecules, the antiparallel molecular arrangement observed in the crystals does not occur in the SAMs. There, the molecules are bonded by the anchoring group (thiolate in the present case) to the substrate and are, thus, arranged in a parallel fashion.

\subsection{Ellipsometry}

The ellipsometric thicknesses of the PP, PPm-down, and PmP-up SAMs were estimated as $1.11 \pm 0.02 \mathrm{~nm}, 0.93 \pm 0.02 \mathrm{~nm}$, and $1.10 \pm 0.03 \mathrm{~nm}$, respectively. As all the molecules mentioned above have a similar length of $\sim 1.0 \mathrm{~nm}$ and the length of $\mathrm{S}-\mathrm{Au}$ bond is estimated as $0.24 \mathrm{~nm},{ }^{75,76}$ it can be assumed that all three thiols form dense monolayers with similar packing densities, even though the PPm-down SAM might be not as closely packed as the two other monolayers. A simple comparison of the derived layer thickness to the sum of the molecular length and the length of the $\mathrm{S}-\mathrm{Au}$ bond permits a rough estimation of the molecular tilt angles $(\beta)$ relative to the surface normal, which amount to $\sim 26^{\circ}$ (PP), $\sim 41^{\circ}$ (PPm-down), and $\sim 26^{\circ}$ (PmP-up).

\subsection{Wetting Properties}

The wetting properties of the PmP-up, PPm-down and PP SAMs are important in the context of OSC growth on SAM-modified electrodes ${ }^{39}$ and as a fingerprint of the decoupling of the SAM-OSC (SAM/ambient) interface and the dipole engineering.

Advancing $\left(\theta_{\text {adv }}\right)$ and receding $\left(\theta_{\text {rec }}\right)$ water contact angles (WCA) for the PmP-up, PPm-down, and PP SAMs prepared from THF and ethanol solutions are presented in Figures $2 \mathrm{a}$ and $2 \mathrm{~b}$, respectively. For the THF preparation, both $\theta_{\mathrm{adv}}$ and $\theta_{\text {rec }}$ show similar values for the different monolayers, verifying the general notion of a decoupling the SAM-OSC (SAM/ambient) interface and the dipole engineering. There is, however, a weak, systematic dependence of WCA on the molecular dipole moment, which means that the influence of the molecular dipole cannot be completely neglected within the given two-rings molecular architecture. Note that, 
generally, the relation between molecular dipole and surface energy represents an interesting issue (see e.g. ref 15).

For the ethanol preparation, $\theta_{\text {adv }}$ and $\theta_{\text {rec }}$ are only similar for the PPm-down and PP SAMs (showing also a good correlation with the WCA values for the THF case), whereas the values for the PmP-up monolayers are noticeably lower, which agrees with the previous measurement performed in context of device applications of these SAMs and is mostly related to the polar component of the surface energy. ${ }^{39}$ The reasons for this behavior are unclear at the moment, since we could not observe any other distinct differences between the films prepared from THF and ethanol.

Note that the WCA values for the reference PP SAMs are higher than those published in literature $\left(\theta_{\text {adv }}\right.$ and $\theta_{\text {rec }}$, viz. $73^{\circ}$ and $\left.69^{\circ}\right),{ }^{40}$ indicative of the very high quality of our films.

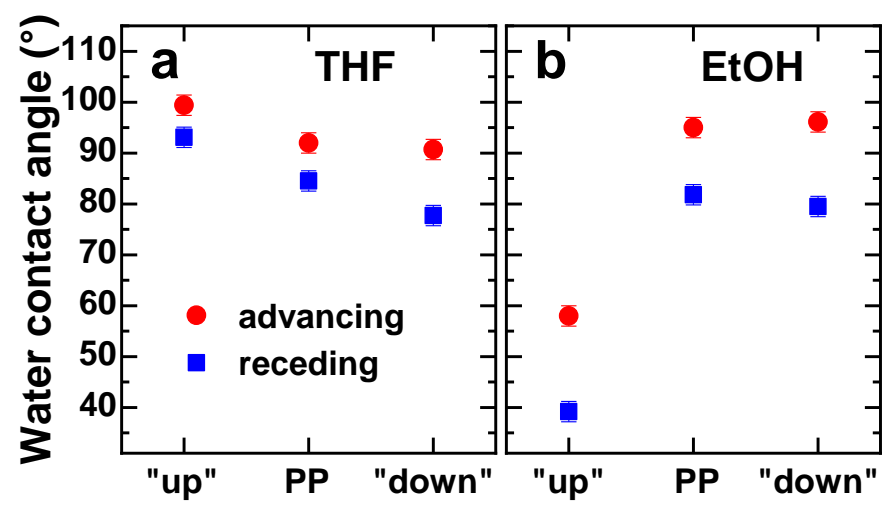

Figure 2. Advancing (red circles) and receding (blue squares) water contact angles for the PmPup ("up"), PPm-down ("down") and PP SAMs prepared from THF (a) and ethanol (b).

\subsection{STM}

Molecular packings in the PP, PPm-down, and PmP-up SAMs were characterized by STM. Representative large scale and high resolution STM images of these monolayers are shown in Figure 3. For the PmP-up SAM, well-ordered structures could be obtained in a straightforward manner after an immersion time of $24 \mathrm{~h}$. The domains have a size of about $20 \mathrm{~nm}$ and expose the well-known $(\sqrt{3} \times \sqrt{3}) \mathrm{R} 30^{\circ}$ superstructure corresponding to a packing density of $4.63 \times 10^{14}$ molecules $/ \mathrm{cm}^{2}$. The situation was more complex for the PPm-down monolayers, for which domains were only observed after immersion times of at least seven days. The domains with a size of about 4-5 nm were still quite disordered after this time, consistent with the lower apparent film thickness determined in the ellipsometry experiments. When the immersion time was prolonged to twelve days, the average domain size increased to $8 \mathrm{~nm}$ and the order within the domains improved. $A(\sqrt{ } 3 \times \sqrt{ } 3) \mathrm{R} 30^{\circ}$ unit cell could be observed. 


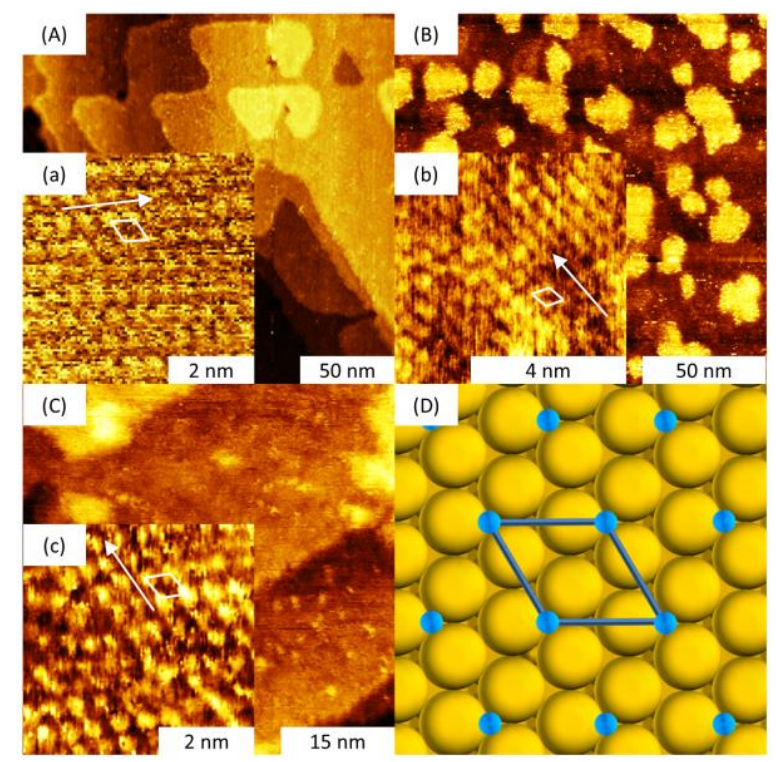

Figure 3. Large scale (A, B, C) and high magnification (a, b, c) STM images of the dominant $(\sqrt{3} \times \sqrt{3}) R 30^{\circ}$ phase in the PmP-up (A, a), PP (B, b) and PPm-down (C, c; after 8 days of immersion) SAMs along with a scheme of the respective molecular arrangement (D; for the sketch we arranged the sulfur on top of a three-fold hollow side). In panels $a, b$, and c the unit cell and the $\langle\mathbf{1 1} \overline{\mathbf{2}}\rangle$ direction (white arrows) are marked. Parameters: (A) $1.0 \mathrm{pA}, 200 \mathrm{mV}$; (a) 12.0 pA, $220 \mathrm{mV}$; (B) $1.5 \mathrm{pA}, 200 \mathrm{mV}$; (b) $20.0 \mathrm{pA}, 60 \mathrm{mV}$; (C) $5.0 \mathrm{pA}, 200 \mathrm{mV}$; (c) $40.0 \mathrm{pA}$, $200 \mathrm{mV}$.

Note that the PmP-up, PPm-down, and PP SAMs do not induce pronounced etch pits on $\mathrm{Au}(111)$ as typical for some thiol-based monolayers (see e.g. ref 77) but rather form Au islands as seen in the large scale images in Figure 3. Such a behavior is frequently observed if the anchoring $\mathrm{S}$ atom is directly attached to the aromatic system (see e.g. ref 59).

Note also that the $(\sqrt{ } 3 \times \sqrt{ } 3) \mathrm{R} 30^{\circ}$ structure was recorded as the dominant structural phase for the reference PP SAMs as well but it was difficult to obtain well-resolved images of this arrangement. In accordance with literature data, ${ }^{59}$ this issue is presumably related to the small size of the crystalline domains and some polymorphism. This can generally be explained by a mismatch of the optimal molecular packing and the underlying (111) lattice of the gold substrate ${ }^{78}$ in particular, the preferred packing of bulk biphenyl deviates slightly from a hexagonal arrangement. ${ }^{79}$

In addition to the $(\sqrt{3} \times \sqrt{ } 3) \mathrm{R} 30^{\circ}$ structure, a $(2 \times 2)$ phase was also observed in all three monolayers. Such a structure has, in fact, been reported previously for the PP SAMs prepared 
on $\mathrm{Au}(111)$ by vapor deposition. ${ }^{80}$ This phase is characterized by a $\sim 25 \%$ lower packing density compared to the $(\sqrt{3} \times \sqrt{3}) \mathrm{R} 30^{\circ}$ structure. In view of the XPS data (vide infra) it is, however, believed to be the minority phase and the $(\sqrt{3} \times \sqrt{3}) \mathrm{R} 30^{\circ}$ structure is considered as reference structure throughout the entire paper. In passing we note that the STM data do not provide information on the relative orientation of neighboring molecules. I.e., it cannot be deduced whether the molecules pack in a cofacial or in a herringbone structure, where the latter, due to the presence of two symmetry-inequivalent molecules, would require a larger unit cell than $(\sqrt{ } 3 \times \sqrt{3}) \mathrm{R} 30^{\circ}$. This issue will be addressed later, when modeling the structural properties of the investigated SAMs.

\subsection{XPS}

S 2p, N 1s, and C 1s XP spectra of the PmP-up, PPm-down, and PP SAMs are presented in Figure 4; the complementary $\mathrm{Au} 4 \mathrm{f}$ and $\mathrm{O} 1 \mathrm{~s}$ spectra are compiled in Figure $\mathrm{S} 12$ in the Supporting Information.

The S 2p XP spectra of all three films in Figure 4a exhibit a single $S$ 2p $3 / 2,1 / 2$ doublet at $~ 162.0$ $\mathrm{eV}\left(\mathrm{S} 2 \mathrm{p}_{3 / 2}\right)$. The BE is representative of the thiolate species bound to noble metal substrates, ${ }^{46}$ indicative of the distinct SAM character of all the studied films. The intensity of the doublet is similar over the series, being only slightly weaker for PmP-up/Au. This suggests a similar packing density of all three SAMs. This conclusion is supported by the similar intensities of the $\mathrm{Au} 4 \mathrm{f}$ signal, determined via the attenuation by the SAM overlayer (Figure S12a in the Supporting Information).

The N 1s XP spectra of PmP-up/Au and PPm-down/Au are presented in Figure 4b. These spectra exhibit a single $\mathrm{N}$ 1s peak assigned to the nitrogen atoms in the pyrimidine ring of the SAM precursors. Consequently, the spectrum of PP/Au does not show any $\mathrm{N} 1 \mathrm{~s}$ signal, in accordance with the chemical composition of the SAM. The BEs of the N 1s peak are, however, distinctly different for the PPm-down and PmP-up SAMs, being $398.05 \mathrm{eV}$ and $398.65 \mathrm{eV}$, respectively. This difference cannot stem exclusively from the different screening of the photoemission hole by the substrate, resulting in a slightly lower $\mathrm{N} \mathrm{1s} \mathrm{BE} \mathrm{value} \mathrm{for} \mathrm{PPm-}$ down/ $\mathrm{Au}$, since the respective effect is not that strong, and can be estimated as $0.05-0.1 \mathrm{eV}$ in the given case. ${ }^{81}$ Rather, it is a consequence of electrostatically induced XPS shifts, ${ }^{16,17,70,82,83}$ as shall be discussed in section 3.10. 


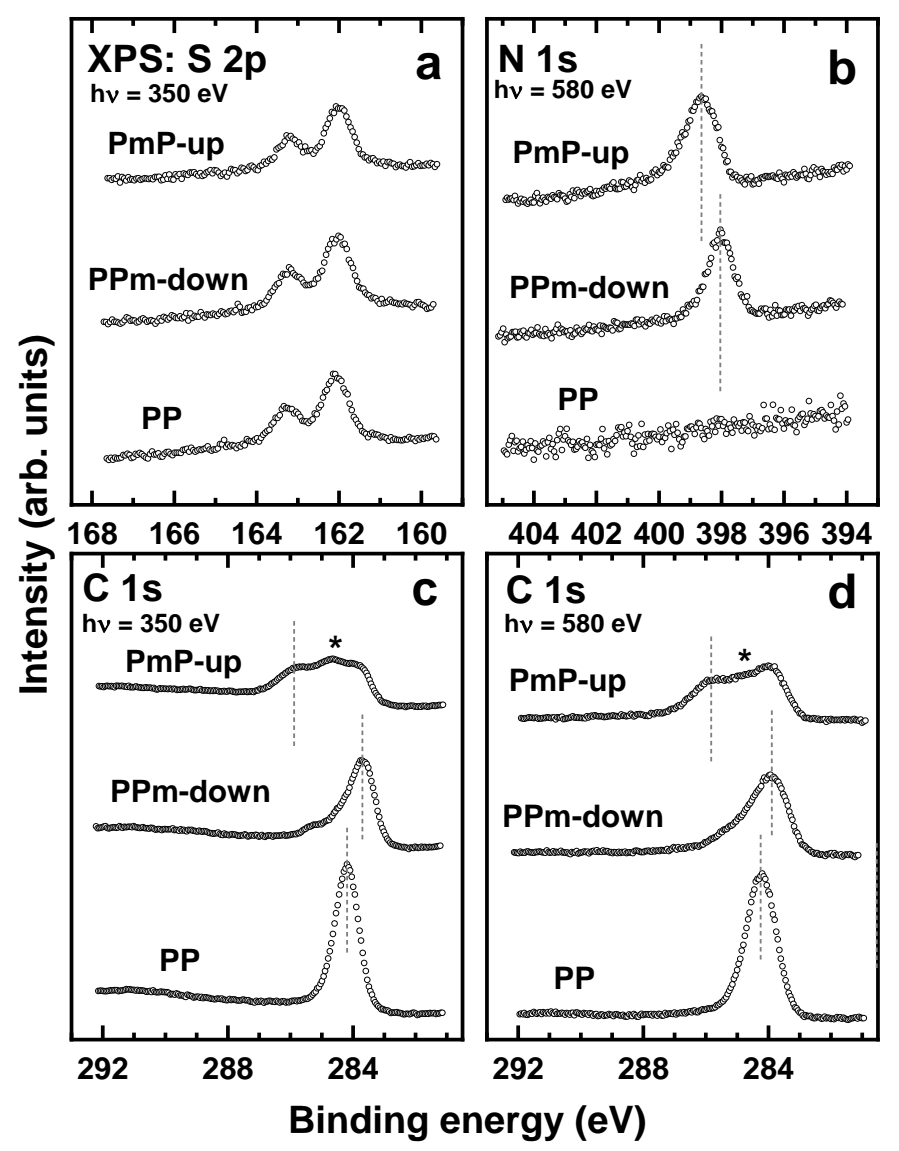

Figure 4. S 2p (a), N 1s (b), and C 1s (c, d) XP spectra of the PmP-up, PPm-down, and PP SAMs. The spectra were acquired at photon energies of $350 \mathrm{eV}(\mathrm{a}, \mathrm{c})$ and $580 \mathrm{eV}(\mathrm{b}, \mathrm{d})$. The vertical dashed lines are guides to the eye. A feature with a strongly photon-energy dependent intensity between the two major peaks in the spectrum of PmP-up is marked by *.

The C 1s XP spectra of PmP-up/Au and PPm-down/Au are presented in Figures 4c and 4d. They are distinctly different and cannot be explained by a mere superposition of a pyrimidine spectrum and a phenylene spectrum, as both SAMs consist of these two rings. Rather, the spectra are again strongly influenced by electrostatic effects, associated with the periodic arrangement of the dipole moments of the embedded pyrimidine moieties. ${ }^{17,70,82,83}$ These effects will be discussed in detail below, when analyzing the electronic structure of the SAMs (section 3.10), relying on a comparison of our experiments with DFT simulations and on an analysis of literature data on related compounds. They are also mostly responsible for the larger apparent width of the XPS features in PmP-up. Beyond that, a direct comparison between calculated and measured spectra for both systems (see the Supporting Information) yields a minor additional broadening in PmP-up, hinting towards a slightly reduced order in these films. 
Along with the qualitative analysis of the XPS data, their quantitative evaluation was performed (see section 2 for details), yielding the same packing density of ca. $4.5 \times 10^{14}$ molecules $/ \mathrm{cm}^{2}$ for both monolayers together with effective thicknesses of $0.93 \pm 0.05 \mathrm{~nm}$ and $1.00 \pm 0.05 \mathrm{~nm}$ for the PPm-down and PmP-up SAMs, respectively. Significantly, the values for the PPm-down and

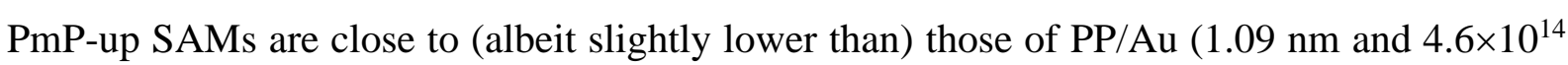
molecules $/ \mathrm{cm}^{2}$, corresponding to the $(\sqrt{3} \times \sqrt{ } 3) \mathrm{R} 30^{\circ}$ structure; see section 2$)$. This suggests a similar molecular packing with some disorder due to the presence of the dipolar pyrimidine groups (or the occurrence of $(2 \times 2)$ structures as a minority phase, as discussed in section 3.5 ).

\subsection{IRRAS}

IRRAS offers a means to characterize the molecular identity as well as the orientation of surface bound species. In Figure 5, the IR spectra of the bulk PP, PPm-down and PmP-up materials as well as the IRRA spectra of the corresponding SAMs are displayed, along with the results of DFT calculations on isolated molecules.

In Table 1 the most prominent vibrational modes for the PPm-down and PmP-up monolayers are compiled and assigned, based on the DFT calculations. Additionally, the TDMs of these modes with respect to the molecular backbone and the molecular plane (assuming a close-toplanar conformation) are given as "\|" (parallel), “ $\perp$ " (perpendicular, in plane), and "oop" (perpendicular, out of plane). From Figure 5 and Table 1 it is evident that in the IRRA spectra those bands are attenuated whose TDMs are perpendicular to the molecular axes, while the other bands stay intense. According to the surface selection rules for metal substrates, ${ }^{84}$ this indicates an almost upright molecular orientation in all studied SAMs. Apart from this qualitative conclusion, the tilt angle $\beta$ (deviation of the direction of the molecular backbone from the surface normal; see Figure 6) for the PmP-up and PPm-down molecules on the gold substrate were estimated using the method established by Parikh and Allara. ${ }^{85}$ This method compares the relative intensities of three IR bands (each with an independent TDM) from the IR spectra of the SAM and the neat substance. For the PmP-up and PPm-down monolayers the intensities of bands $2\left(\right.$ oop), $7(\|)$ and $8(\perp)$ were compared. This yields tilt angles of $24^{\circ} \pm 11^{\circ}$ and $14^{\circ} \pm 10^{\circ}$, respectively. Note that the given approach in principle allows also evaluating the twist angle $\gamma$ (rotation around the molecular axis; see Figure 6) but the low intensities of the $\perp$ and oop bands in the IRRA spectra cause very large error bars for this parameter, so that we refrain from presenting the respective results. Note also that the low intensities of these bands 
prevent the evaluation of the tilt and twist angles for PP. Complementary data on molecular orientation were obtained using NEXAFS spectroscopy (vide infra).
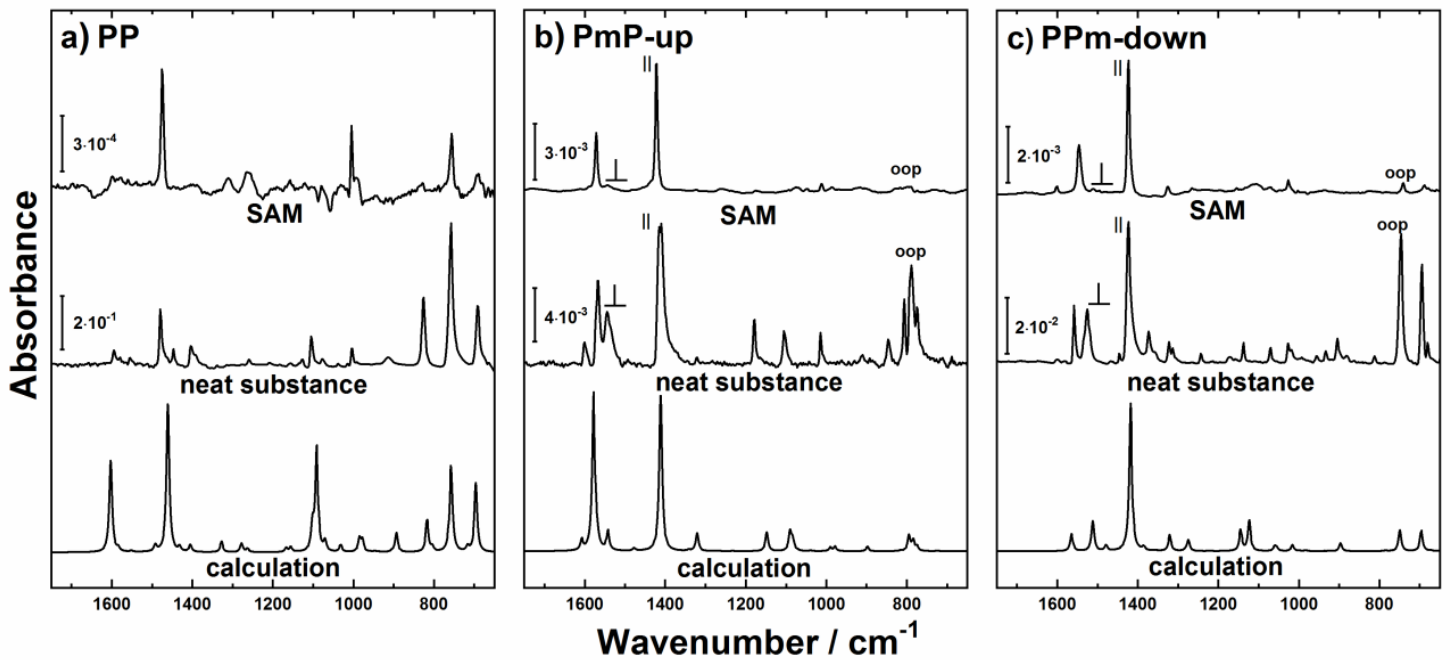

Figure 5. IRRA spectra of PP (a), PmP-up (b), and PPm-down (c) SAMs (upper curves) along with the IR spectra of the neat substances (middle curves) and the DFT calculated spectra (bottom curves). Absorbance scale bars are given for the experimental spectra while the calculated spectra are displayed in arbitrary units. The modes, which were used for the calculation of the tilt and twist angles, are labeled as "I|", “ $\perp$ " and "oop". Note that the selected " $\perp$ " mode, which only has reasonable intensity for the PmP-up and PPm-down case, is partly merged with a "\|" mode appearing at a higher wavenumber.

Table 1. Positions (given in $\mathrm{cm}^{-1}$ ) of the most important vibrational modes in the IR spectra of the PPm-down and PmP-up molecules and corresponding SAMs, together with the respective theoretical values (DFT). The assignment of the vibrational modes was carried out on the basis of the DFT calculations, which also provided the orientations of the respective TDMs.

\begin{tabular}{|c|c|c|c|c|c|c|c|c|}
\hline \multirow[b]{2}{*}{ No. } & \multirow[b]{2}{*}{ Mode ${ }^{a, b)}$} & \multirow[b]{2}{*}{$\mathrm{TDM}^{\mathrm{c})}$} & \multicolumn{3}{|c|}{ PPm-down } & \multicolumn{3}{|c|}{ PmP-up } \\
\hline & & & DFT & Neat $^{d)}$ & $\mathrm{SAM}^{\mathrm{d})}$ & DFT & Neat $^{\text {d) }}$ & $\mathrm{SAM}^{\mathrm{d})}$ \\
\hline 1 & $\gamma \mathrm{CH}$ ring twist & oop & 696 & $695 \mathrm{~s}$ & $688 w$ & & & \\
\hline 2 & $\gamma \mathrm{CH}$ ring twist & oop & 749 & $747 \mathrm{~s}$ & $741 w$ & 794 & $789 \mathrm{~s}$ & $790 w$ \\
\hline 3 & $v \mathrm{CC}, \delta \mathrm{NCN}$ & $\|$ & & & & 979 & $1014 \mathrm{~m}$ & 1012 w \\
\hline 4 & $\delta \mathrm{CH}$ & $\|$ & & & & 1148 & $1179 \mathrm{~m}$ & $1175 \mathrm{vw}$ \\
\hline 5 & $v \mathrm{CC}, \delta \mathrm{NCN}$ & $\|$ & & & & 1321 & 1322 w & 1324 w \\
\hline 6 & $v \mathrm{CC} \mathrm{CN}, \delta \mathrm{CH}$ & $\perp$ & 1386 & $1373 \mathrm{~m}$ & & & & \\
\hline 7 & $v \mathrm{CN}^{(\mathrm{s})}$ & $\|$ & 1418 & 1424 vs & 1424 vs & 1412 & 1410 vs & 1422 vs \\
\hline 8 & $v \mathrm{CN}^{\text {(as) }}$ & $\perp$ & 1512 & $1526 \mathrm{~m}$ & 1511 w & 1542 & $1544 \mathrm{~m}$ & 1544 w \\
\hline 9 & $\delta \mathrm{CH}, v \mathrm{CN}$ & $\|$ & 1565 & $1558 \mathrm{~m}$ & $1546 \mathrm{~m}$ & 1578 & $1567 \mathrm{~s}$ & $1572 \mathrm{~m}$ \\
\hline 10 & $v \mathrm{SH}$ & & 2604 & $2535 \mathrm{~m}$ & & 2617 & $2578 \mathrm{vw}$ & \\
\hline
\end{tabular}

\footnotetext{
a) Due to the differences in the molecular structure of PPm-down and PmP-up, the equivalents of some strong bands in the spectrum of one of the molecules have very low intensities in the spectrum of the other molecule and can become almost invisible. Where this applies, we refrain from displaying their wavenumbers; b) $v$ : stretch mode, $\delta$ : in plane bending mode, $\gamma$ : out of plane bending mode, s: symmetric, as: asymmetric; ${ }^{\mathrm{c})} \|$ : parallel to molecular backbone, $\perp$ : perpendicular to main molecular backbone and in plane of the aromatic ring, oop: perpendicular to the aromatic plane; ${ }^{\mathrm{d})} \mathrm{vs:}$ very strong, s: strong, m: medium, w: weak, vw: very weak.
} 


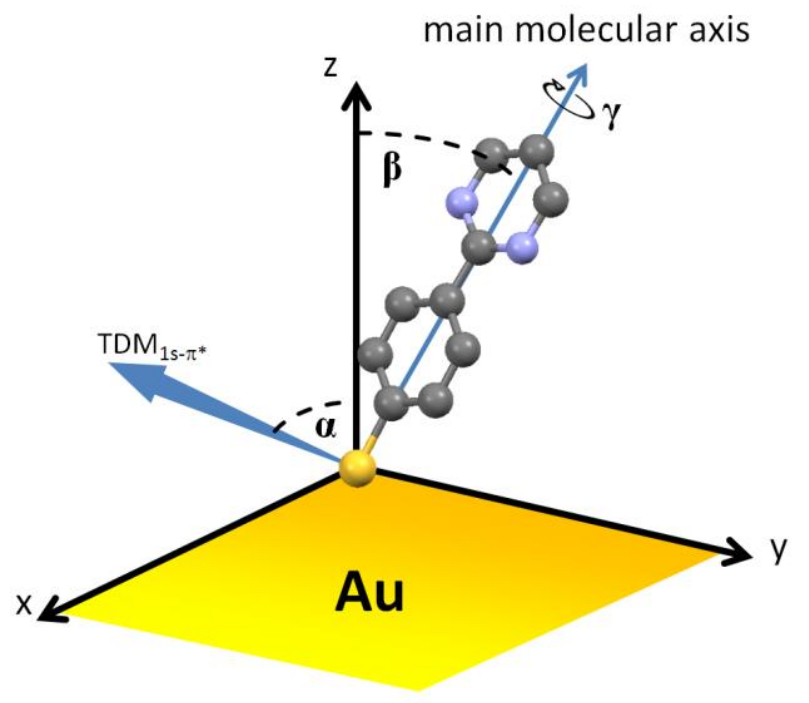

Figure 6. Exemplary illustration of PmP-up in a coordinate system along with $\beta$ (tilt angle of the main molecular axis with respect to the surface normal), $\gamma$ (molecular twist angle), and $\alpha$ (tilt angle of the $\pi^{*}$ orbitals: see section 3.8). Both aromatic rings are considered to be coplanar consistent with the results of the simulations (see below) and X-ray diffraction analysis for the single crystals (see section 3.2). The twist angle is defined such that it is zero when the tilt occurs perpendicular to the molecular plane. The transition dipole moment of the $1 \mathrm{~s}-\pi^{*}$ resonance lies then in the plane spanned by the $\mathrm{z}^{-}$and the main molecular axis.

\subsection{NEXAFS Spectroscopy}

$\mathrm{C}$ and N K-edge NEXAFS data for the PmP-up and PPm-down SAMs are presented in Figures 7 and 8, respectively; the reference data for PP/Au are compiled in Figure S13 in the Supporting Information. In Figures 7 and 8 two kinds of spectra are depicted. First, there are spectra acquired at an X-ray incidence angle of $55^{\circ}$ (magic angle), which are representative of the electronic structure of the films (unoccupied molecular orbitals) and are not affected by orientational effects. ${ }^{61}$ Second, there are curves corresponding to the difference between the spectra collected under the normal $\left(90^{\circ}\right)$ and grazing $\left(20^{\circ}\right)$ incidence geometry. These curves are useful fingerprints of orientational order and molecular orientation, ${ }^{61}$ showing the extent of the resonance intensity change upon variation of the angle between the electric field vector of the primary X-ray beam and the TDMs of the relevant molecular orbitals. 


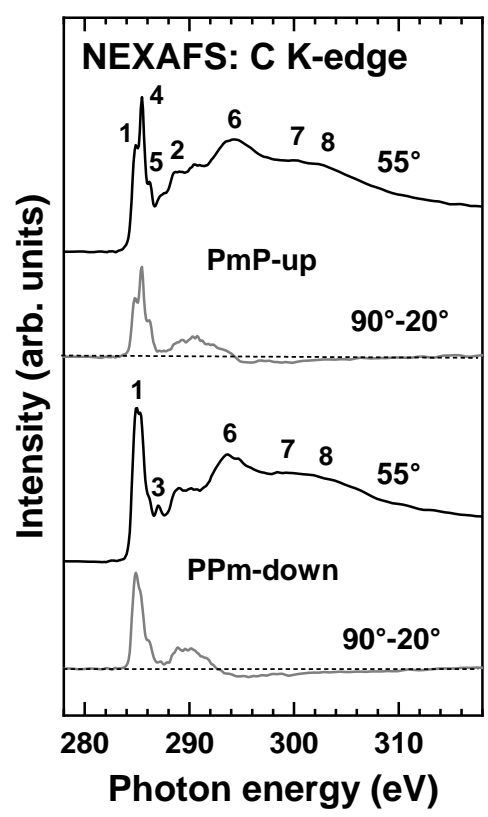

Figure 7. C K-edge NEXAFS spectra of the PmP-up and PPm-down SAMs acquired at an Xray incidence angle of $55^{\circ}$ (black lines), along with the respective difference between the spectra collected under the normal $\left(90^{\circ}\right)$ and grazing $\left(20^{\circ}\right)$ incidence geometry (gray lines). Individual absorption resonances are marked by numbers (see text for the assignments). The horizontal dashed lines correspond to zero.

Let us first consider the $55^{\circ}$ spectra, starting with the $\mathrm{C}$ K-edge ones. Within the generally acceptable building block scheme in X-ray absorption spectroscopy ${ }^{61}$ the spectra of PmPup/Au and PPm-down/Au can be understood as superpositions of the component spectra of phenyl and pyrimidine rings. Generally, the spectrum of phenyl and oligophenyl SAMs is dominated by the strong $\pi_{1}{ }^{*}$ resonance at $\sim 285.0 \mathrm{eV}(\mathbf{1})$, accompanied by the respective $\pi_{2}{ }^{*}$ peak (2) at $\sim 288.8 \mathrm{eV}$ and several broader $\sigma^{*}$ resonances (6-8) at higher excitation energies (see Figure S13 in the Supporting Information). ${ }^{44,61,86,87}$ In addition, in oligophenyl SAMs a $\mathrm{R}^{*} / \mathrm{C}-\mathrm{S}^{*}$ resonance $(3)$ at $\sim 287.0 \mathrm{eV}$ is frequently observed. ${ }^{44}$ The spectrum of pyrimidine is dominated by a complex feature, with maxima at 284.7-285.1 eV (close to 1; low intensity), $285.4 \mathrm{eV}$ (4; high intensity), and $285.86 \mathrm{eV}$ (5; high intensity), containing contributions from several $\pi^{*}$ resonances. ${ }^{88,89}$ The spectrum exhibits also a variety of comparably weak $\pi^{*}$ and Rydberg resonances as well as broader $\sigma^{*}$ resonances at higher excitation energies. ${ }^{88,89}$

The above features are indeed observed in the $55^{\circ} \mathrm{C}$ K-edge NEXAFS spectra of PmP-up/Au and PPm-down/Au in Figure 7, with the weights modulated by the attenuation effects for the PEY signal ${ }^{90}$ and a certain decrease in intensity of absorption resonances for the moieties located far away from the SAM-ambient interface. ${ }^{44}$ Accordingly, the spectrum of PPm- 
down/Au, having the phenyl ring in the top position and the pyrimidine ring in the bottom position (Figure 1), is dominated by the features of the phenyl moiety, with just a "broadening" of the most intense $\pi_{1}{ }^{*}$ resonance (1) due to the admixture of the pyrimidine resonances $\mathbf{4}$ and 5. In contrast, the spectrum of $\mathrm{PmP}-\mathrm{up} / \mathrm{Au}$, having the pyrimidine ring in the top position and phenyl ring in the bottom position (Figure 1), is dominated by the pyrimidine resonances 4 and 5 forming a complex absorption structure in the pre-edge region, with a contribution of the $\pi_{1}{ }^{*}$ resonance (1) of the phenyl ring. At the same time, both for PPm-down/Au and PmP-up/Au, the post-edge region is dominated by the $\sigma^{*}$ resonances of phenyl (compare with the data for PP/Au in Figure S13 in the Supporting Information) which are generally more distinct than those of pyrimidine.

The $55^{\circ} \mathrm{N}$ K-edge NEXAFS spectra of PPm-down/Au and PmP-up/Au in Figure 8 exhibit the characteristic features of pyrimidine. ${ }^{88,89}$ The spectra are dominated by a strong $\pi^{*}$ resonance at $\sim 398.5 \mathrm{eV}$ (1) accompanied by a mixed $\pi^{*}$-Rydberg feature at $402.7 \mathrm{eV}$ (2) and Rydberg features at higher excitation energies (3 and 4). The intensity of the resonances is somewhat lower for PPm-down/Au, which is related to the stronger attenuation of the PEY signal for the pyrimidine ring in the bottom position as well as to the slightly lower coverage as compared to PmP-up/Au (see sections 3.3 and 3.6).

While the $55^{\circ}$ NEXAFS spectra allow to verify the chemical identity of the PPm-down and PmP-up SAMs, the difference $90^{\circ}-20^{\circ}$ spectra in Figures 7 and 8 give insight into the molecular orientation in the films. In these spectra, pronounced difference peaks at the positions of the absorption resonances are observed, indicative of a high orientational order. Further, the peaks associated with the $\pi^{*}$ resonances are positive, whereas those related to the $\sigma^{*}$ resonances are negative. Considering that the TDMs of the $\pi^{*}$ and $\sigma^{*}$ orbitals are directed perpendicular and parallel to the ring planes, respectively, the signs of the difference peaks mean a largely upright molecular orientation in the SAMs, in qualitative agreement with the ellipsometry, IR spectroscopy, and XPS results (see sections 3.3, 3.6 and 3.7). 


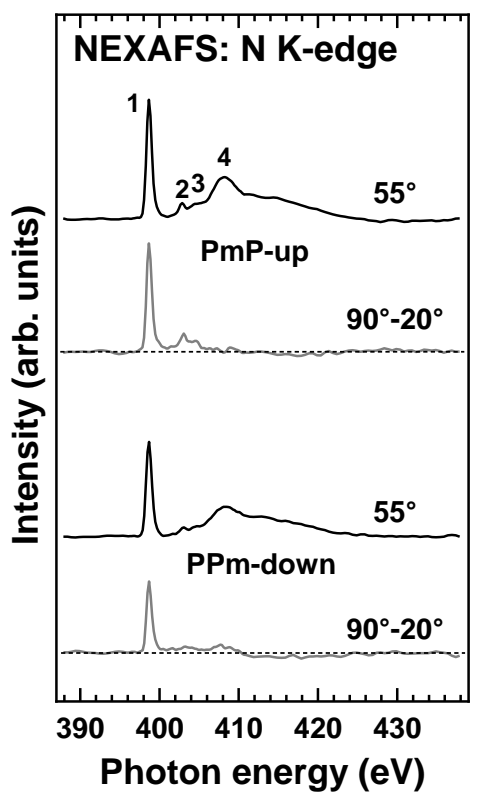

Figure 8. N K-edge NEXAFS spectra of the PmP-up and PPm-down SAMs acquired at an Xray incidence angle of $55^{\circ}$ (black lines), along with the respective difference between the spectra collected under the normal $\left(90^{\circ}\right)$ and grazing $\left(20^{\circ}\right)$ incidence geometry (gray lines). Individual absorption resonances are marked by numbers (see text for the assignments). The horizontal dashed lines correspond to zero.

In addition to the above-described qualitative analysis, a quantitative evaluation of the entire set of the NEXAFS data for PPm-down/Au and PmP-up/Au was performed, relying on the most prominent $\pi^{*}$ resonances at the $\mathrm{C}$ and $\mathrm{N}$ K-edge and using the standard theoretical framework for the vector-like orbitals, suitable in the given case. ${ }^{61}$ Within the respective evaluation procedure, the intensity of a particular $\pi^{*}$ resonance was derived from the spectra, plotted as a function of the X-ray incidence angle and fitted by the theoretical curves for a vector-like orbital, with the average tilt angle of the $\pi^{*}$ orbital with respect to the surface normal, $\alpha$ (see Figure 6), being the only parameter. ${ }^{44,91}$ The derived values of this parameter for the $\mathrm{C}$ and $\mathrm{N}$ $\mathrm{K}$-edge resonances are compiled in Table 2 . The very similar values obtained evaluating the $\mathrm{C}$ and $\mathrm{N}$ K-edge resonances suggest that the two rings are coplanar or close-to-coplanar in the SAMs, since the derived angles are predominantly (C K-edge) or exclusively (N K-edge) representative of the orientation of the phenyl and pyrimidine rings, respectively. Furthermore, it is consistent with the simulations discussed in the following section and with the very small, respectively, vanishing inter-ring torsions seen in the single crystal data for the precursor materials in section 3.2 . 
Table 2. Average tilt angles of the $\pi^{*}$ orbitals in the PmP-up and PPm-down SAMs, calculated on the basis of the C K-edge and N K-edge NEXAFS data. The accuracy of the values is $\pm 3^{\circ}$.

\begin{tabular}{cccc} 
Monolayer & $\begin{array}{c}\text { Tilt angle }(\alpha) \text { from } \\
\text { the C K-edge data }\end{array}$ & $\begin{array}{c}\text { Tilt angle }(\alpha) \text { from } \\
\text { the N K-edge data }\end{array}$ & $\begin{array}{c}\text { Average value } \\
\text { PmP-up/Au }\end{array} 6^{\circ}$ \\
PPm-down/Au & $65.6^{\circ}$ & $69.1^{\circ}$ & $67.5^{\circ}$ \\
\hline
\end{tabular}

Based on the average tilt angles of the $\pi^{*}$ orbitals, average molecular tilt angles with respect to the surface normal, $\beta$, can be calculated using $\cos \alpha=\sin \beta \times \cos \gamma$, where $\gamma$ is the molecular twist angle (see Section 3.7). The latter angle cannot be determined from the NEXAFS data (except for a specially derivatized molecules) ${ }^{72}$. Thus, it is usually derived from bulk structures, IR data, or from simulations. In the present case, we will rely on the latter (see next section), taking the level of agreement between the experimental and theoretical values of $\alpha$ as a criterion for the reliability of the simulations. Provided that this agreement is satisfactory, molecular tilt and twist angles can then be directly determined from the simulated structures.

\subsection{Modeling the Structural Properties of the SAMs}

The optimized geometries of the PmP-up and PPm-down SAMs are shown in Figure 9. As mentioned in the methodology section, all SAMs were modeled using a $(3 \mathrm{x} \sqrt{3})$ rect unit cell containing two molecules, which yields the same packing density as the $(\sqrt{3} \times \sqrt{3}) \mathrm{R} 30^{\circ}$ structure $\left(4.6 \times 10^{14}\right.$ molecules $/ \mathrm{cm}^{2}$; in line with the value observed experimentally). It, however, allows for a herringbone arrangement of the molecules. To determine the actual film structure, we then compared the energetics of the herringbone and cofacial arrangements of the molecules and found the former to be more stable in both studied systems (by $0.12 \mathrm{eV}$ per molecule for PmP$\mathrm{up} / \mathrm{Au}$ and by $0.11 \mathrm{eV}$ for $\mathrm{PPm}-\mathrm{down} / \mathrm{Au}$ ). The individual molecules assume a planar conformation (with inter-ring torsions below $3^{\circ}$ for PmP-up/Au and $7^{\circ}$ for $\mathrm{PPm}-\mathrm{down} / \mathrm{Au}$ ) consistent with the experimental results discussed above. A detailed analysis of the intermolecular interactions causing this specific intra- and intermolecular arrangement is provided in ref 92 for the reference PP/Au system. 

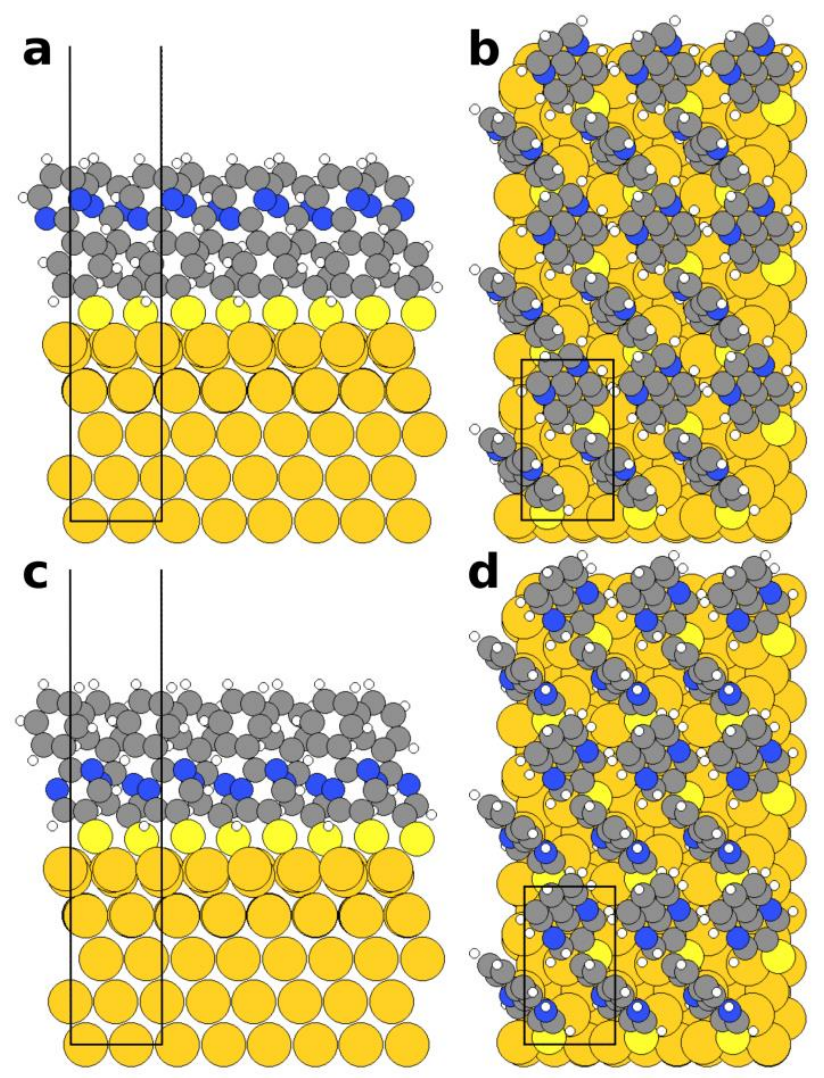

Figure 9. DFT-optimized structures of the PmP-up (a,b) and PPm-down (c,d) SAMs. Color code: $\mathrm{H}$ - white, $\mathrm{C}$ - grey, $\mathrm{N}$ - blue, $\mathrm{S}$ - light yellow, and $\mathrm{Au}$ - dark yellow.

From the simulated geometries, the structural parameters can be directly obtained and compared to the experimental ones. The derived tilt angles of the $\pi^{*}$ orbitals, $\alpha$, the molecular tilt angles, $\beta$, and the molecular twist angles, $\gamma$, are reported in Table 3, where the subscripts 1 and 2 refer to the symmetry inequivalent molecules in the unit cell. For $\alpha$ also the value averaged over the two molecules, $\alpha_{\mathrm{av}}$, is given. To be consistent with the NEXAFS evaluation, it is obtained via $\cos ^{2} \alpha_{\mathrm{av}}=\left(\cos ^{2} \alpha_{1}+\cos ^{2} \alpha_{2}\right) / 2$, since the tilt angle enters the formula for the intensity of an absorption resonance associated with a vector-like orbital as $\cos ^{2} \alpha .{ }^{61}$

The calculated average tilt angles of the $\pi^{*}$ orbitals agree very well with the values obtained in the NEXAFS experiments (see Table 2), which underlines the reliability of the theoretical modelling and makes the respective $\beta$ values reasonable estimates for the molecular tilt in the studied SAMs. The calculated tilt angle for the PPm-down SAM is somewhat larger than for the PmP-up monolayer. This agrees with the ellipsometry (section 3.3) and XPS (section 3.6) data, which suggest higher effective thickness in the PmP-up case (corresponding to a smaller molecular tilt). The non-negligible differences in the $\alpha$ values between the two molecules in the unit cell are not due to different tilts, but result from strongly different twists (caused by 
different directions into which the molecules tilt). Therefore, they can be considered as a consequence of the herringbone-arrangement of the molecules.

Table 3. DFT calculated average tilt angles of the $\pi^{*}$ orbitals $\alpha$, molecular tilt angles $\beta$ and molecular twist angles $\gamma$ in the PmP-up and PPm-down SAMs. 1, and 2 denote the values for the two symmetry inequivalent molecules in the unit cell. The value of $\alpha_{\mathrm{av}}$ is obtained employing the averaging described in the main text. The twist angle is defined such that it is zero when the tilt occurs perpendicular to the molecular plane. ${ }^{72}$

\begin{tabular}{cccccccc} 
Monolayer & $\alpha_{\text {av }}$ & $\alpha_{1}$ & $\alpha_{2}$ & $\beta_{1}$ & $\beta_{2}$ & $\gamma_{1}$ & $\gamma_{2}$ \\
\hline PmP-up/Au & $69.5^{\circ}$ & $75.8^{\circ}$ & $64.6^{\circ}$ & $29.6^{\circ}$ & $25.6^{\circ}$ & $60.2^{\circ}$ & $7.7^{\circ}$ \\
PPm-down $/ \mathrm{Au}$ & $66.5^{\circ}$ & $75.7^{\circ}$ & $59.5^{\circ}$ & $31.5^{\circ}$ & $30.8^{\circ}$ & $61.8^{\circ}$ & $7.2^{\circ}$
\end{tabular}

\subsection{Electronic Properties of the SAMs - Simulated XP Spectra}

As a first step to analyze the electronic structure of the SAMs, we calculated their XP corelevel spectra. They are shown in the top panel of Figure 10 for a signal damping consistent with a primary photon energy of $580 \mathrm{eV}$. As simulations within the initial-state approach rely on the calculation of the Kohn-Sham orbital energies of the core levels, they provide information only on the relative energetics of the different atoms. ${ }^{93-96}$ Therefore, the values have been rigidly shifted to higher binding energies (BEs) by $18.94 \mathrm{eV}$ (a value we typically observe for SAMs), such that the experimental and calculated spectra of the "parent" PP SAM are aligned.

The overall shape of the spectra agrees well with the experiments (see Figures $4 \mathrm{c}$ and $4 \mathrm{~d}$ ): The spectrum of the PP SAM is characterized by a single peak with a binding energy of $284.2 \mathrm{eV}$ (in general agreement with the experimental data ${ }^{46,97}$. Also for PPm-down/Au the spectrum is clearly dominated by a single feature, which is, however, shifted to lower binding energies by ca. $0.6 \mathrm{eV}$. Moreover, there is a pronounced tail at the high binding-energy side of the spectrum with a shoulder at ca. $284.8 \mathrm{eV}$. In contrast, in the $580 \mathrm{eV}$ spectrum of PmP-up/Au, two more clearly separated main features, split by $1.4 \mathrm{eV}$, can be identified, with the more intense feature essentially coinciding with the main peak for the PP SAM. Interestingly, for smaller primary photon energies $(350 \mathrm{eV})$ in the experiments, a third feature appears between the two peaks of the $580 \mathrm{eV}$ spectra (see Figures $4 \mathrm{c}$ and $4 \mathrm{~d}$ ), which is also seen in the corresponding simulations, albeit with a reduced relative intensity (see the simulated spectrum of PmP-up/Au for $350 \mathrm{eV}$ contained in the Supporting Information, Figure S14). 


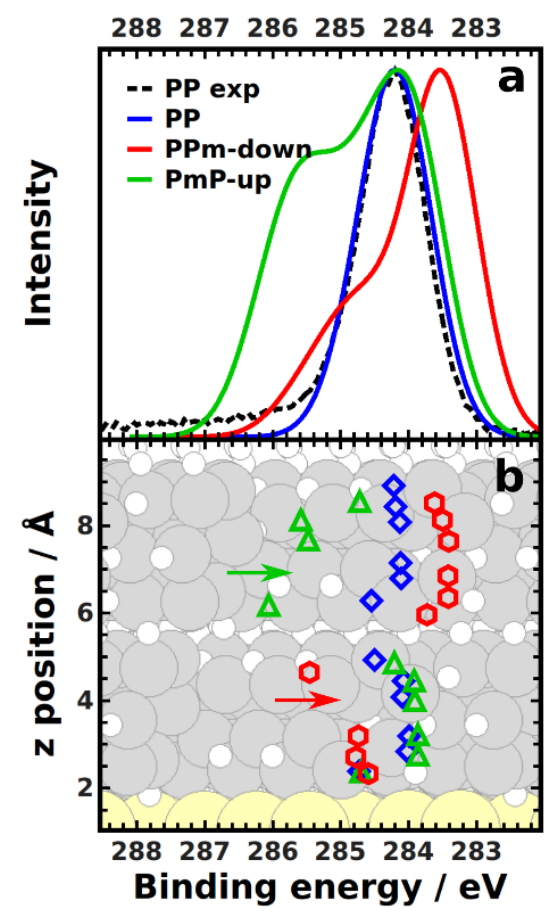

Figure 10. Top: DFT calculated XP spectra for the PP (blue), the PPm-down (red) and the PmP-up (green) SAMs. The spectra have been aligned to the experimental spectrum of PP and a damping consistent with a primary photon energy of $580 \mathrm{eV}$ was assumed (for details see the main text, respectively, the Supporting Information). Bottom: atom-resolved, (shifted) corelevel binding energies of individual $\mathrm{C}$ atoms in the PP (blue diamonds), PPm-down (red hexagons) and PmP-up (green triangles) SAMs. The energies are averaged over equivalent atoms in the two molecules contained in the unit cell. The PP SAM shown in the background serves to identify the $\mathrm{z}$-position (vertical axis) of individual $\mathrm{C}$ atoms. The $\mathrm{z}$ axis is perpendicular to the substrate, with its origin corresponding to the position of the image plane (i.e., $0.9 \AA$ above the average $\mathrm{z}$ position of the topmost Au layer, see the Supporting Information). The average positions of the $\mathrm{N}$ atoms in the PPm-down and PmP-up SAMs are indicated by red and green arrows, respectively. For both spectra and energies of the individual levels, screening effects by the substrate according to the electrostatic model described in the Supporting Information have been considered.

A distinct advantage of the calculated XP spectra is that it is straightforward to decompose them into contributions from individual $\mathrm{C}$ atoms. This is done based on the bottom panel of Figure 10, which shows the core level energies for all $\mathrm{C}$ atoms of the three systems considered here. For the case of PP/Au, the core-levels of most of the $\mathrm{C}$ atoms are located at comparable energies. The small, continuous shift to higher BEs with increasing the distance to the substrate is attributed to differences in screening. Only the positions of the $\mathrm{C}$ atom bonded to the thiolate and of the two $\mathrm{C}$ atoms forming the bridge between the two rings are somewhat shifted to even 
higher BEs. This can be attributed to their different chemical environments (chemical shifts) and, especially for the lowest $\mathrm{C}$ atom, also to particularly large screening effects by the substrate.

The situation becomes much more complex for the pyrimidine containing systems. There, one encounters a superposition of strong chemical shifts (due to $\mathrm{C}$ atoms bonded to the $\mathrm{N}$ atoms $)^{98}$ and electrostatic shifts due to the pyrimidine dipoles. The origin of the latter shifts are so-called collective electrostatic effects caused by a parallel alignment of dipoles. ${ }^{99-101}$ This results in a sharp step in the electrostatic energy at the position of the dipole layer, which shifts the corelevel energies relative to the Fermi level for all atoms further away from the substrate than that dipole layer. As the Fermi level of the substrate serves as the reference energy for the XPS experiment, the consequence is a change in the respective core-level binding energies. ${ }^{70}$ Notably, this shift is not related to any change in the local charge density around the said atoms, as it is observed for chemically identical species solely separated by a dipole layer; ${ }^{16,17}$ it is also not related to the evanescent field of the dipole assembly as the decay length of that field is extremely short. ${ }^{99}$

For PPm-down/Au, such electrostatic shifts caused by the pyrimidine dipoles result in a reduction of the $\mathrm{BEs}$ of the $\mathrm{C}$ atoms of the upper ring compared to the $\mathrm{PP} / \mathrm{Au}$ reference system in spite of the identical atomic charges on the outermost rings found for both systems (see the Supporting Information). In contrast, the BEs of the $\mathrm{C}$ atoms in the bottom pyrimidine ring are increased compared to PP/Au (with the exception of the atom bonded to S). This is due to chemical shifts, as all these atoms are bonded to electronegative $\mathrm{N}$ atoms. These chemical shifts reach a maximum for the topmost $\mathrm{C}$ atom of that ring, which is bonded to two $\mathrm{N}$ atoms. Consequently, the low-lying $\mathrm{C}$ atoms are responsible for the high-BE tail in the spectra, which is consistent with the experimental observation that the intensity of that tail decreases for primary photon energies of $350 \mathrm{eV}$.

For PmP-up/Au, the core-level energies of the bottom ring are hardly shifted relative to the $\mathrm{PP} / \mathrm{Au}$ reference, as these $\mathrm{C}$ atoms do not experience any extra electrostatic shifts (the only dipoles between them and the metal are those associated with the dipoles of the thiolate groups present in all monolayers). Additionally, compared to $\mathrm{PPm}-\mathrm{down} / \mathrm{Au}$, none of the respective $\mathrm{C}$ atoms experiences a chemical shift. Conversely, the core-levels of the $\mathrm{C}$ atoms in the pyrimidine ring are strongly shifted to higher BEs. This can be understood as a superposition of a chemical shift (due to the bonding to the $\mathrm{N}$ atoms) and an electrostatic shift (originating from the pyrimidine dipole). As both shifts go in the same direction for PmP-up/Au, the associated BEs 
are particularly large, which explains the pronounced high-BE peak observed for this system both in the experiments and in the simulations. Interestingly, the core-level of the topmost $\mathrm{C}$ atom in the PmP-up SAM is found at a smaller BE, as it is not directly bonded to the nitrogen atoms. Therefore, we hold this atom responsible for the peak at $284.6 \mathrm{eV}$ visible in the experimental $350 \mathrm{eV}$ spectrum (Figure 4c; where the features of the lower-lying $\mathrm{C}$ atoms are attenuated due to the very small escape depth of the photoelectrons).

The above-described different combinations of chemical and electrostatic shifts in PmP-up/Au and PPm-down/Au explain both the different lineshapes of the XPS spectra and the different binding energies of the main peak.

The above-discussed electrostatic shifts are also responsible for the different core-level BEs of the $\mathrm{N}$ atoms. Qualitatively consistent with the experiments, we calculated the $\mathrm{N} 1 \mathrm{~s}$ energy of $\mathrm{PPm}$-down/Au to be $0.5 \mathrm{eV}$ smaller than for PmP-up/Au. As the chemical environments of the $\mathrm{N}$ atoms are essentially identical in both systems, the reason for the shift is that the $\mathrm{N}$ atoms are located on different "sides" of the primary components of the differently oriented pyrimidine dipoles.

\subsection{Electronic Properties of the SAMs - Measured and Calculated SAM-Induced Work Function Changes}

The parallel alignment of the pyrimidine dipoles also causes a shift in the sample work function. This is shown for the PmP-up, PPm-down, and PP SAMs in Figures 11a and 11b for films grown from THF and ethanol, respectively. Significantly, the THF values are only slightly smaller than the ethanol ones. This suggests that the ability of the PmP-up and PPm-down films to manipulate charge carrier injection barriers in organic electronics assemblies does not depend significantly on the solvent applied for their preparation. Also, the values presented here for the SAM prepared from ethanol on high-quality, commercial gold substrates (see section 2) are very close to those for "technical gold substrates" (a RMS value of $1.8 \mathrm{~nm}$ over a scan area of $4 \times 4 \mu \mathrm{m}^{2}$ ) treated analogous to electrodes patterned device structures (see ref 39 for details); viz. the value for PmP-up/Au is identical and that for PPm-down/Au is only slightly higher (by $\sim 0.1 \mathrm{eV}$ ). This means that the dipole engineering by the PmP-up and PPm-down SAMs is sufficiently robust with respect to the character of the substrate. 


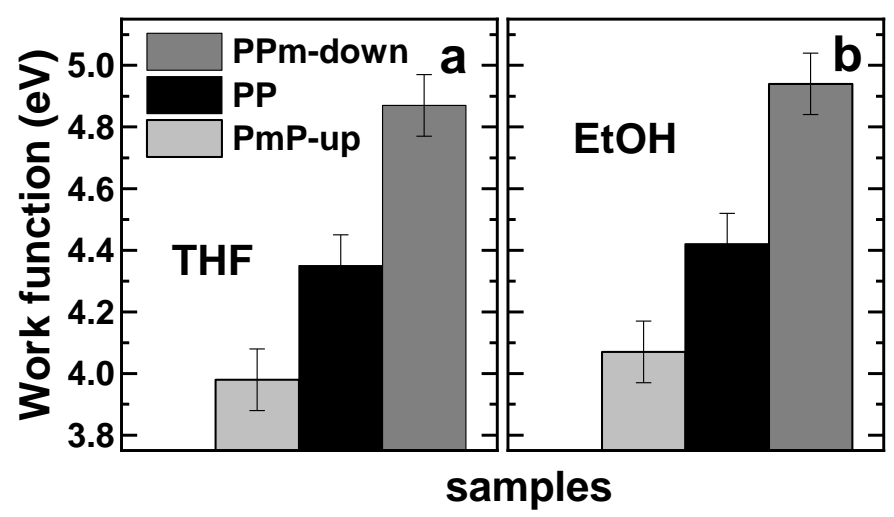

Figure 11. Work function values for the PmP-up, PPm-down, and PP SAMs prepared from THF (a) and ethanol (b) measured with a Kelvin probe on high-quality, commercial Au substrates (see section 2). The gray scale code is given in the panels.

In line with the orientation of the dipole moment of the embedded pyrimidine group, the work functions of PPm-down/Au and PmP-up/Au are higher (by $+0.52 \mathrm{eV}$ ), respectively lower (by $-0.37 \mathrm{eV}$ ), than for PP/Au (THF preparation). The observed WF shifts are very close to those observed for the previously reported terphenylmethanethiol-based SAMs, also containing a single pyrimidine unit per molecule (see Figure $S 1$ in the Supporting Information), viz. +0.56 $\mathrm{eV}$ and $-0.42 \mathrm{eV} .{ }^{19}$ The slightly larger shifts in the latter systems can in part be explained by a somewhat smaller molecular inclination, which results in a larger projection of the dipole moment onto the surface normal, which defines the SAM-induced change of the work function.

The work function difference between PPm-down/Au and PmP-up/Au is $\sim 0.9 \mathrm{eV}$, where we expect that the work function can be adjusted continuously within this range by mixing PPmdown and PmP-up molecules in a single SAM, as it has been demonstrated for a variety of different mixed SAMs ${ }^{7,23,102-104}$ including systems with embedded dipoles ${ }^{20-22}$.

The experimental trend is confirmed by the simulations with a decrease of the work function for PmP-up/Au and an increase for PPm-down/Au (compared to the reference PP SAM). The absolute magnitude of the effect $( \pm 0.65 \mathrm{eV})$ is, however, overestimated, which is in line with what we have observed for a variety of other systems, especially other pyrimidine-containing compounds. ${ }^{19}$ This is in part a consequence of the simulations assuming a densely packed periodic structure, with the entire film in a $(3 \times \sqrt{3})$ rect arrangement. Such a model does not account for disordered regions like grain boundaries ${ }^{19}$ or the patches of lower-coverage films with molecules arranged in a $(2 \times 2)$ pattern, as recorded in the STM measurements (see section 3.5). Additionally, we observe that both the dipole moment of the pyrimidine unit and the bonddipole associated with the thiolate docking group depend on the used methodology (i.e., there 
is an inevitable systematic error associated with using a specific functional like PBE; see the Supporting Information for more details). Interestingly, the calculated work function changes for $\mathrm{PPm}$-down/Au and PmP-up/Au relative to PP/Au are identical in the simulations, while they somewhat differ in the experiments. This could again be a consequence of disorder within the experimentally studied SAM, as discussed in the Supporting Information.

\subsection{Electrical Conductance}

Besides the changes of the electrode work function, also the electrical conductance of the monolayer is a crucial parameter for the application of the SAMs as interfacial layers between the electrode and the active layer in organic electronic devices. Semilogarithmic current-density versus voltage plots for $\mathrm{Au} / \mathrm{SAM} / / \mathrm{EGaIn}$ junctions with the PmP-up, PPm-down, and PP (reference) SAMs are presented in Figure 12; they agree well with the analogous data measured within the previous device study. ${ }^{39}$ The currents are higher by ca. one order of magnitude than those observed for the analogous terphenylmethanethiol-based molecules with embedded pyrimidine group (see Figure S1 in the Supporting Information). ${ }^{39,105}$ This indicates superior electric transport properties of the PmP-up and PPm-down monolayers, making these SAMs particularly promising for applications in organic electronics and photovoltaic devices. Such a stronger coupling in the absence of a methyl spacer has been found in various experiments ${ }^{106,107}$ and is also consistent with the massively broadened transmission functions calculated for thiolbonded SAMs. ${ }^{108,109}$

An interesting side aspect are quite similar current values for the SAMs with opposite directions of the dipole moment. This is consistent with the observation for terphenylmethanethiol-based SAMs containing central pyrimidine units at varying orientation, where a careful statistical analysis of the current-voltage curves yielded a rectification of only 1.2. ${ }^{110}$ Also, alkyl SAMs including polar groups either into the backbones or as tail groups did not yield significant rectification ratios. ${ }^{111}$ What is somewhat surprising for the present systems is that increasing the coupling to the Au electrode by skipping the methyl linker does not have a distinct impact on rectification, considering that asymmetries of the coupling to the two electrodes have been predicted to yield more sizable rectification ratios in related systems. ${ }^{112}$ 


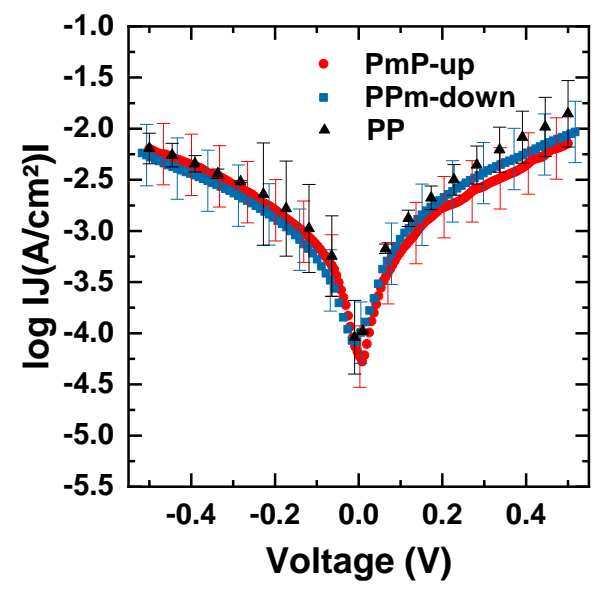

Figure 12. Semilogarithmic current-density versus voltage plots for Au/SAM//EGaIn junctions with the PmP-up (red circles), PPm-down (blue squares), and PP (black triangles) SAMs.

\subsection{Applications in Organic Thin Film Transistors}

Due to their structural and electrostatic properties, the PmP-up and PPm-down SAMs can be used in organic electronics and photovoltaics, providing energy level adjustment at the interfaces between the electrodes and OSC or between the electrodes and buffer layers. The application of these monolayers in organic thin film transistors (OTFTs) and related electronic circuits on solid (glass) and flexible (polycarbonate plastic film) substrates is described in detail in our recent dedicated study. ${ }^{39}$ To illustrate the potential of the PmP-up and PPm-down SAMs, Figure 13 shows the output curves of both p-type (pentacene as OSC) and n-type (C60 as OSC) OTFTs featuring SAM-modified Au source and drain electrodes. The curves in Figure 13 show a clear effect of the embedded dipole. For pentacene-based devices, the currents increase by a factor of 25 when employing PPm-down SAMs compared to the PmP-up case, while the trend is reversed for the $\mathrm{C}_{60}$ devices with the ratio here even exceeding a factor of 60 . This can be associated with the contact resistance changing by more than two orders of magnitude ${ }^{39}$ for electrodes modified with the two SAMs. Generally, PPm-down favors hole and PmP-up favors electron injection. This is fully consistent with the changes in the respective injection barriers ${ }^{39}$ arising from the work function modifications described in section 3.11. 


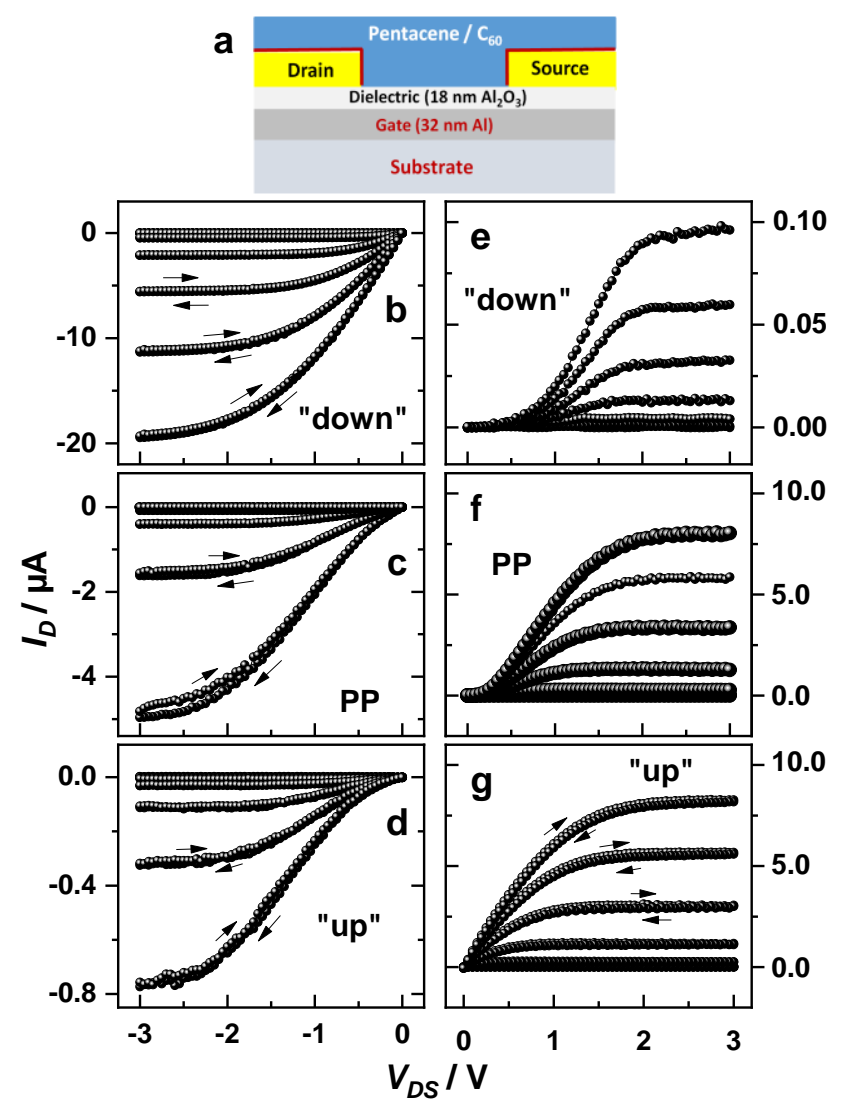

Figure 13. Schematic of the bottom gate, bottom contact OTFTs (a) featuring SAM-modified source and drain gold electrodes $(50 \mathrm{~nm})$ and either pentacene or $\mathrm{C}_{60}$ as OSC. The SAMs are indicated by red line. The description of the fabrication process and the technical details can be found elsewhere. ${ }^{39}$ Panels (b)-(d) show typical output characteristics of p-type pentacene OTFTs with the electrodes modified by the PPm-down (b; "down"), PP (c), and PmP-up (d; "up") SAMs; panels (e)-(g) contain typical output characteristics of n-type $\mathrm{C}_{60}$ OTFTs with the electrodes modified by the PPm-down (e; "down"), PP (f) and PmP-up (g; "up") SAMs. The curves were acquired at several different $\mathrm{V}_{\mathrm{GS}}$ values varied in 0.5 steps from 0.5 to $-2.5 \mathrm{~V}$ (b), from 1 to $-2 \mathrm{~V}$ (c), from 1.2 to $-1.8 \mathrm{~V}$ (d), from 3.4 to $6.4 \mathrm{~V}$ (e), and from 2.1 to $5.1 \mathrm{~V}$ (f and g). The different $V_{G S}$ ranges for the different OTFTs are a consequence of the different onset voltages $\left(\mathrm{V}_{\mathrm{on}}\right)$ observed in the different devices. In this way, the values of $\mathrm{V}_{\mathrm{GS}}-\mathrm{V}_{\text {on }}$ (defining the output current of OTFTs) are varied similarly for either p-type or n-type devices. Accordingly, the output characteristics become directly comparable. The OTFTs were fabricated either on glass (p-type) or polycarbonate plastic film (n-type). Adapted from ref 39.

\subsection{Summary of the Results}


The basic structural parameters of the PP, PPm-down, and PmP-up monolayers derived by ellipsometry, XPS, STM, IR, and NEXAFS spectroscopy experiments as well as by the quantum mechanical simulations are compiled in Table 4. The values obtained with the different techniques correlate well with each other, even though there is a certain scattering of individual parameters. The effective thicknesses and packing densities of the PP, PPm-down, and PmP-up SAMs are similar, suggesting similar molecular packing. The packing density values correlate well with the packing density of the $(\sqrt{3} \times \sqrt{3}) \mathrm{R} 30^{\circ}$ structure, which was indeed directly observed by STM (Figure 3), even though the imaging was not easy and a certain polymorphism was recorded. The molecular conformation in the PPm-down and PmP-up monolayers is essentially planar, as suggested by the bulk crystallography (Figures S9 and S11 in the Supporting Information) and the NEXAFS spectroscopy data and additionally confirmed by the DFT simulations (Figure 9). The simulations favor a herringbone molecular arrangement (Figure 9), which requires a $(3 \times \sqrt{3})$ rect unit cell, which however has the same packing density and is compatible with the $(\sqrt{3} \times \sqrt{3}) \mathrm{R} 30^{\circ}$ structure. The simulations also shed light on the molecular orientation in the PPm-down and PmP-up SAMs (Table 3), with a very good agreement with the results of the NEXAFS spectroscopy (Table 2) and with the IR-derived value for PmP-up/Au. The particularly small tilt calculated from the IR data for PPm-down/Au appears less realistic and is presumably related to a low intensity of specific vibration modes used for the evaluation (see section 3.7). Insights into the electronic structure of the SAMs could be gained from comparing calculated and measured XPS data (Figures 4 and 10) revealing a superposition of electrostatic and chemical shifts of the core-level binding energies. We also observed a good correlation between the KP data (Figure 11) and calculated electrostatic properties of the PPm-down and PmP-up SAMs. The electric conductance of these monolayers is found to be rather high (Figure 12), which is a sizable advantage for their applications in organic electronics and photovoltaics. The potential of the PmP-up and PPm-down SAMs in organic electronics is illustrated by representative experimental data for the bottom gate, bottom contact OTFTs, featuring SAM-modified source and drain gold electrodes (Figure 13).

Table 4. Basic parameters of the PP, PmP-up, and PPm-down SAMs derived from the experimental data and theoretical simulations. 


\begin{tabular}{|c|c|c|c|c|c|c|c|c|}
\hline \multirow{2}{*}{$\begin{array}{l}\text { Monolayer } \\
\text { Method }\end{array}$} & \multicolumn{2}{|c|}{ Effective thickness [nm] } & \multirow{2}{*}{$\begin{array}{l}\text { Packing density } \\
\text { [molecules } \mathbf{~ c m}^{-2} \text { ] } \\
\text { XPS }\end{array}$} & \multicolumn{4}{|c|}{ Molecular tilt angle $\left[^{\circ}\right]$} & \multirow{2}{*}{$\begin{array}{c}\text { Twist } \\
\text { angle }\left[{ }^{\circ}\right] \\
\text { theory }\end{array}$} \\
\hline & XPS & ellipsometry & & ellipsometry & NEXAFS & IR & theory & \\
\hline PP & $1.09 \pm 0.05$ & $1.11 \pm 0.02$ & $4.6 \times 10^{14}$ & $26 \pm 10$ & $19 \pm 3^{b)}$ & n.d. & $27.1 / 24.7$ & $56.1 / 13.1$ \\
\hline PmP-up & $1.0 \pm 0.05$ & $1.10 \pm 0.03$ & $4.5 \times 10^{14}$ & $26 \pm 10$ & $29 \pm 3^{\text {a) }}$ & $24 \pm 11$ & $29.6 / 25.6$ & $60.2 / 7.7$ \\
\hline PPm-down & $0.93 \pm 0.05$ & $0.93 \pm 0.02$ & $4.5 \times 10^{14}$ & $41 \pm 10$ & $31.5 \pm 3^{\mathrm{a})}$ & $14 \pm 10$ & $31.5 / 30.8$ & $61.8 / 7.2$ \\
\hline
\end{tabular}

a) The NEXAFS-derived tilt angles for PmP-up/Au and PPm-down/Au were calculated from the average values given in Table 2, assuming the twist angles from Table 3 and the same tilt angles of both nonequivalent molecules in the unit cell. For the simulations, the tilt and twist angles for both nonequivalent molecules in the unit cell are given. ${ }^{\text {b) }}$ The NEXAFS-derived tilt angle for PP/Au was calculated assuming a twist angle of $32^{\circ}$ taken from the bulk crystal data. ${ }^{79,113}$

\section{Conclusions}

Based on the concept of the embedded dipoles in molecular self-assembly on solid substrates, we designed and synthesized optimal molecules for controlling the energy level alignment at interfaces between electrode and organic semiconductor layers. The molecules, PmP-up and PPm-down, feature the thiol docking group and a short heteroaromatic backbone consisting of a non-polar phenyl ring and a polar pyrimidine group. The latter is "embedded" in two opposite orientations in a way that its nitrogen atoms are buried with respect to the SAM-substrate and SAM-ambient interfaces. This decouples these interfaces from the dipole engineering. The resulting dipolar systems have significant inherent flexibility and are relatively easy to modify as the key functionality of the SAM is maintained when changing, e.g., the docking group (now thiol to bind to noble metals) or when adjusting the tail-group polarity and reactivity (now inert $\mathrm{C}-\mathrm{H})$.

In view of the popularity of gold as substrate when studying SAMs and as electrode material in organic electronics, we fabricated and characterized in detail PmP-up, PPm-down, as well as reference non-polar PP monolayers on $\mathrm{Au}(111)$ substrates, putting particular emphasis on the electronic properties. The results of a variety of complementary spectroscopic and microscopic techniques consistently suggest the formation of densely packed and well-defined SAMs, with all molecules bonded to the substrate via the thiolate docking group in an almost upright geometry. Packing density, molecular orientation, structure and wetting properties of these SAMs were found to be nearly independent of their electrostatic properties (with only one exception; see section 3.4). The latter correlate precisely with the molecular architecture and the orientation of the embedded dipolar group. More insights into the electrostatic properties of the SAM can be obtained by comparing XP spectra with calculated core-level binding-energies, which reveals pronounced shifts due to collective electrostatic effects on top of chemical shifts. Concomitantly, depending on the pyrimidine orientation, the SAMs studied here can change 
the Au work function by $\sim 0.9 \mathrm{eV}$. Moreover, currents through the studied SAMs measured in EGaIn junctions are relatively high. These aspects place the PmP-up and PPm-down SAMs as ideal systems for model studies in the context of interfacial dipole engineering but also as highly suitable functional blocks for device applications. The latter is illustrated by realizing highly promising $\mathrm{p}$ - and n-type transistors using Au as electrode material, while optimizing the level alignment through chemisorbing SAMs containing suitably oriented pyrimidine groups.

\section{Associated content}

\section{Supporting Information}

The Supporting Information is available free of charge on the ACS Publication website at DOI: Exact description of the procedures for the synthesis of the SAM precursors; crystallographic data; additional spectroscopic data; computational details (PDF).

\section{Author Information}

\section{Corresponding Author}

*E-mail: egbert.zojer@tugraz.at

*E-mail: aterfort@chemie.uni-frankfurt.de

*E-mail: Michael.Zharnikov@urz.uni-heidelberg.de

\section{ORCID}

Egbert Zojer: 0000-0002-6502-1721

Andreas Terfort: 0000-0003-2369-5151

Michael Zharnikov: 0000-0002-3708-7571

\section{Notes}

The authors declare no competing financial interest.

\section{Acknowledgements}

E.S. and M.Z thank the Helmholtz Zentrum Berlin for the allocation of synchrotron radiation beamtime at BESSY II and A. Nefedov and Ch. Wöll for the technical cooperation during the experiments there. The work was financially supported by the German Research Foundation 
(Deutsche Forschungsgemeinschaft; DFG) via grants ZH 63/22-1 (E.S. and M.Z.) and TE247/15-1 (M.G. and A.T.) as well as by the Austrian Science Fund (FWF) via grants I2081N20 and J4145-N30. The computational results have been in part achieved using the Vienna Scientific Cluster (VSC3). We thank Albemarle (formerly Rockwood), Frankfurt, and AlzChem AG, Trostberg, for generous gifts of organolithium reagents and 2-hydroxypyrimidine, respectively. 


\section{References}

(1) Koch, N. Energy Levels at Interfaces between Metals and Conjugated Organic Molecules. J. Phys.: Condens. Matter 2008, 20, 184008.

(2) Liu, C.; Xu, Y.; Noh, Y.-Y. Contact Engineering in Organic Field-Effect Transistors. Mater. Today 2015, 18, 79-96.

(3) Casalini, S.; Bortolotti, C. A.; Leonardi, F.; Biscarini, F. Self-Assembled Monolayers in Organic Electronics. Chem. Soc. Rev. 2017, 46, 40-71.

(4) Kymissis, I.; Dimitrakopoulos, C. D.; Purushothaman, S. High-Performance Bottom Electrode Organic Thin-Film Transistors. IEEE Trans. Electron Devices 2001, 48, 10601064.

(5) Bock, C.; Pham, D. V.; Kunze, U.; Käfer, D.; Witte, G.; Wöll, Ch. Improved Morphology and Charge Carrier Injection in Pentacene Field-Effect Transistors with ThiolTreated Electrodes. J. Appl. Phys. 2006, 100, 114517.

(6) Acton, O.; Dubey, M.; Weidner, T.; O’Malley, K. M.; Kim, T.-W.; Ting, G. G.;

Hutchins, D.; Baio, J. E.; Lovejoy, T. C.; Gage A. H.; et. al., Simultaneous Modification of Bottom-Contact Electrode and Dielectric Surfaces for Organic Thin-Film Transistors Through Single-Component Spin-Cast Monolayers. Adv. Funct. Mater. 2011, 21, 1476-1488.

(7) Kim, J.; Rim, Y. S.; Liu, Y.; Serino, A. C.; Thomas, J. C.; Chen, H.; Yang, Y.; Weiss, P. S. Interface Control in Organic Electronics Using Mixed Monolayers of Carboranethiol Isomers. Nano Lett. 2014, 14, 2946-2951.

(8) Ulman, A. Formation and Structure of Self-Assembled Monolayers. Chem. Rev. 1996, 96, 1533-1554.

(9) Love, J. C.; Estroff, L. A.; Kriebel, J. K.; Nuzzo, R. G.; Whitesides, G. M. SelfAssembled Monolayers of Thiolates on Metals as a Form of Nanotechnology. Chem. Rev. 2005, 105, 1103-1169.

(10) De Boer, B.; Hadipour, A.; Mandoc, M. M.; Van Woudenbergh, T.; Blom, P. W. M. Tuning of Metal Work Functions with Self-Assembled Monolayers. Adv. Mater. 2005, 17, $621-625$.

(11) Hamadani, B. H.; Corley, D. A.; Ciszek, J. W.; Tour, J. M.; Natelson, D. Controlling Charge Injection in Organic Field-Effect Transistors Using Self-Assembled Monolayers. Nano Lett. 2006, 6, 1303-1306.

(12) Heimel, G.; Romaner, L.; Brédas, J. L.; Zojer, E. Interface Energetics and Level Alignment at Covalent Metal-Molecule Junctions: $\pi$-Conjugated Thiols on Gold. Phys. Rev. Lett. 2006, 96, 196806. 
(13) Heimel, G.; Romaner, L.; Zojer, E.; Bredas, J.-L. The Interface Energetics of SelfAssembled Monolayers on Metals. Acc. Chem. Res. 2008, 41, 721-729.

(14) Lange, I.; Reiter, S.; Pätzel, M.; Zykov, A.; Nefedov, A.; Hildebrandt, J.; Hecht, S.; Kowarik, S.; Wöll, C.; Heimel G. et. al., Tuning the Work Function of Polar Zinc Oxide Surfaces using Modified Phosphonic Acid Self-Assembled Monolayers. Adv. Funct. Mater. 2014, 24, 7014-7024.

(15) Lee, H. Ju; Jamison, A. C.; Lee, T. R. Surface Dipoles: A Growing Body of Evidence Supports Their Impact and Importance. Acc. Chem. Res. 2015, 48, 3007-3015.

(16) Cabarcos, O. M.; Shaporenko, A.; Weidner, T.; Uppili, S.; Dake, L. S.; Zharnikov, M.; Allara, D. L. Physical and Electronic Structure Effects of Embedded Dipoles in SelfAssembled Monolayers: Characterization of Mid-Chain Ester Functionalized Alkanethiols on Au\{111\}. J. Phys. Chem. C 2008, 112, 10842-10854.

(17) Cabarcos, O. M.; Schuster, S.; Hehn, I.; Zhang, P. P.; Maitani, M. M.; Sullivan, N.; Giguère, J.-B.; Morin, J.-F.; Weiss, P. S.; Zojer, E.; et al. Effects of Embedded Dipole Layers on Electrostatic Properties of Alkanethiolate Self-Assembled Monolayers. J. Phys. Chem. C 2017, 121, 15815-15830.

(18) Bruce, R. C.; You, L.; Förster, A.; Pookpanratana, S.; Pomerenk, O.; Lee, H. Ju; Marquez, M. D.; Ghanbaripour, R.; Zenasni, O.; Lee, T. R.; Hacker, C. A. Contrasting Transport and Electrostatic Properties of Selectively Fluorinated Alkanethiol Monolayers with Embedded Dipoles. J. Phys. Chem. C 2018, 122, 4881-4890.

(19) Abu-Husein, T.; Schuster, S.; Egger, D. A.; Kind, M.; Santowski, T.; Wiesner, A.; Chiechi, R.; Zojer, E.; Terfort, A.; Zharnikov, M. The Effects of Embedded Dipoles in Aromatic Self-Assembled Monolayers. Adv. Funct. Mater. 2015, 25, 3943-3957.

(20) Hehn, I.; Schuster, S.; Wächter, T.; Abu-Husein, T.; Terfort, A.: Zharnikov, M.; Zojer, E. Employing X-ray Photoelectron Spectroscopy for Determining Layer Homogeneity in Mixed Polar Self-Assembled Monolayers. J. Phys. Chem. Lett. 2016, 7, 2994-3000.

(21) Sauter, E.; Gilbert, C.-O.; Boismenu-Lavoie, J.; Morin, J.-F.; Zharnikov, M. Mixed Aliphatic Self-Assembled Monolayers with Embedded Polar Group. J. Phys. Chem. C 2017, 121, 23017-23024.

(22) Sauter, E.; Gilbert, C.-O.; Morin, J.-F.; Terfort, A.; Zharnikov, M. Mixed Monomolecular Films with Embedded Dipolar Groups on $\operatorname{Ag}(111)$. J. Phys. Chem. C 2018, $122,19514-19523$. 
(23) Wu, K.-Y.; Yu, S.-Y.; Tao, Y.-T. Continuous Modulation of Electrode Work Function with Mixed Self-Assembled Monolayers and Its Effect in Charge Injection. Langmuir 2009, $25,6232-6238$.

(24) Venkataraman, N. V.; Zürcher, S.; Rossi, A.; Lee, S.; Naujoks, N.; Spencer, N. D. Spatial Tuning of the Metal Work Function by Means of Alkanethiol and Fluorinated Alkanethiol Gradients. J. Phys. Chem. C 2009, 113, 5620-5628.

(25) Holmlin, R. E., Haag, R.; Chabinyc, M. L.; Ismagilov, R. F.; Cohen, A. E.; Terfort, A.; Rampi, M. A.; Whitesides, G. M. Electron Transport through Thin Organic Films in MetalInsulator-Metal Junctions Based on Self-Assembled Monolayers. J. Am. Chem. Soc. 2001, 123, 5075-5085.

(26) Adams, D. M.; Brus, L.; Chidsey, C. E. D.; Creager, S.; Creutz, C.; Kagan, C. R.; Kamat, P. V.; Lieberman, M.; Lindsay, S.; Marcus, R. A.; et al. Charge Transfer on the Nanoscale: Current Status. J. Phys. Chem. B 2003, 107, 6668-6697.

(27) Branchi, B.; Simeone, F. C.; Rampi, M. A. Active and Non-Active Large-Area MetalMolecules-Metal Junctions. In Unimolecular and Supramolecular Electronics II; Metzger, R. M., Ed.; Top Curr. Chem. 313; Springer: Heidelberg, 2012; pp 85-120.

(28) Yang, G.; Liu, G. New Insights for Self-Assembled Monolayers of Organothiols on Au(111) Revealed by Scanning Tunneling Microscopy. J. Phys. Chem. B 2003, 107, 87468759.

(29) Shaporenko, A.; Brunnbauer, M.; Terfort, A.; Grunze, M.; Zharnikov, M. Structural Forces in Self-Assembled Monolayers: Terphenyl-Substituted Alkanethiols on Noble Metal Substrates. J. Phys. Chem. B 2004, 108, 14462-14469.

(30) Azzam, W.; Bashir, A.; Terfort, A.; Strunskus, T.; Wöll, Ch. Combined STM and FTIR Characterization of Terphenylalkanethiol Monolayers on Au(111): Effect of Alkyl Chain Length and Deposition Temperature. Langmuir 2006, 22, 3647-3655.

(31) Simmons, J. G. Generalized Formula for the Electric Tunnel Effect Between Similar Electrodes Separated by a Thin Insulating Film. J. Appl. Phys. 1963, 34, 1793-1803.

(32) Heimel, G.; Romaner, L.; Bredas, J.-L.; Zojer, E. Odd-Even Effects in Self-Assembled Monolayers of $\omega$-(Biphenyl-4-yl)alkanethiols: A First-Principles Study. Langmuir 2008, 24, 474-482.

(33) Bowers, C. M.; Rappoport, D.; Baghbanzadeh, M.; Simeone, F. C.; Liao, K.-C.; Semenov, S. N.; Żaba, T.; Cyganik, P.; Aspuru-Guzik, A.; Whitesides, G. M. Tunneling across SAMs Containing Oligophenyl Groups. J. Phys. Chem. C 2016, 120, 11331-11337. 
(34) Wächter, T.; Weinhardt, L.; Terfort, A.; Zharnikov, M. Pyridine as a Resonantly Addressable Group to Study Electron Transfer Dynamics in Self-Assembled Monolayers, $J$. Phys. Chem. C 2018, 122, 12534-12548.

(35) Blackman, G. L.; Brown, R. D.; Burden, F. R. The Microwave Spectrum, Dipole Moment, and Nuclear Quadrupole Coupling Constants of Pyrimidine. J. Mol. Spectrosc. 1970, 35, 444-454.

(36) Pergolese, B.; Muniz-Miranda, M.; Bigotto, A. SERS Study of the Adsorption of 2mercaptobenzoxazole on Gold Colloidal Nanoparticles. J. Mol. Struct. 2009, 924-926, 559561.

(37) Cui, B.; Chen, T.; Wang, D.; Wan, L. J. In situ STM Evidence for the Adsorption Geometry of Three N-heteroaromatic Thiols on Au(111). Langmuir 2011, 27, 7614-7619.

(38) Partes, C.; Yildirim, C.; Schuster, S.; Kind, M.; Zharnikov, M.; Terfort, A. SelfAssembled Monolayers of Pseudo-C2v-Symmetric, Low-Band Gap Areneoxazolethiolates on Gold Surfaces. Langmuir 2016, 32, 11474-11484.

(39) Petritz, A.; Krammer, M.; Sauter, E.; Gärtner, M.; Nascimbeni, G.; Schrode, B.; Fian, A.; Gold, H.; Cojocaru, A.; Karner-Petritz, E.; et al. Embedded Dipole Self-Assembled Monolayers for Contact Resistance Tuning in p- and n-Type Organic Thin Film Transistors and Flexible Electronic Circuits. Adv. Funct. Mater. 2018, 28, 1804462.

(40) Kang, J. F.; Ulman, A.; Liao, S.; Jordan, R.; Yang, G. H.; Liu, G. Y. Self-Assembled Rigid Monolayers of 4'-substituted-4-mercaptobiphenyls on Gold and Silver Surfaces. Langmuir 2001, 17, 95-106.

(41) X-AREA: Diffractometer Control Program System; Stoe \& Cie: Darmstadt, Germany, 2002.

(42) Sheldrick, G. M. A Short History of SHELX. Acta Crystallogr., Sect. A: Found. Crystallogr. 2008, 64, 112-122.

(43) Chesneau, F.; Zhao, J.; Shen, C.; Buck, M.; Zharnikov, M. Adsorption of Long-Chain Alkanethiols on Au(111) - A Look from the Substrate by High Resolution X-Ray Photoelectron Spectroscopy. J. Phys. Chem. C 2010, 114, 7112-7119.

(44) Frey, S.; Stadler, V.; Heister, K.; Eck, W.; Zharnikov, M.; Grunze, M.; Zeysing, B.; Terfort, A. Structure of Thioaromatic Self-Assembled Monolayers on Gold and Silver. Langmuir 2001, 17, 2408-2415.

(45) Heister, K.; Zharnikov, M.; Grunze, M.; Johansson, L. S. O.; Ulman, A. Characterization of X-Ray Induced Damage In Alkanethiolate Monolayers by High-Resolution Photoelectron Spectroscopy. Langmuir 2001, 17, 8-11. 
(46) Zharnikov, M. High-Resolution X-Ray Photoelectron Spectroscopy in Studies of SelfAssembled Organic Monolayer. J. Electron Spectr. Relat. Phenom. 2010, 178-179, 380-393.

(47) Sauter, E.; Yildirim, C.; Terfort, A.; Zharnikov, M. Adjustment of the Work Function of Pyridine and Pyrimidine Substituted Aromatic Self-Assembled Monolayers by Electron Irradiation. J. Phys. Chem. C 2017, 121, 12834-12841.

(48) Raiber, K.; Terfort, A.; Benndorf, C.; Krings, N.; Strehblow, H. H. Removal of SelfAssembled Monolayers of Alkanethiolates on Gold by Plasma Cleaning. Surf. Sci. 2005, 595, $56-63$.

(49) Frisch, M. J.; Schlegel, H. B.; Scuseria, G. E.; Robb, M. A.; Cheeseman, J. R.; Scalmani, G.; Barone, V.; Mennucci, B.; Petersson, G. A.; Nakatsuji, H.; et al. Gaussian 09, Revision A. 02; Gaussian: Wallingford, CT, USA, 2009.

(50) Perdew, J. Density-Functional Approximation for the Correlation Energy of the Inhomogeneous Electron Gas. Phys. Rev. B 1986, 33, 8822 - 8824.

(51) Becke, A. D. Density-Functional Exchange-Energy Approximation with Correct Asymptotic Behavior. Phys. Rev. A 1988, 38, 3098 - 3099.

(52) Weigend, F.; Ahlrichs, R. Balanced Basis Sets of Split Valence, Triple Zeta Valence and Quadruple Zeta Valence Quality for H to Rn: Design and Assessment of Accuracy. Phys. Chem. Chem. Phys. 2005, 7, $3297-3305$.

(53) Nefedov, A.; Wöll, C. Advanced Applications of NEXAFS Spectroscopy for Functionalized Surfaces, in Surface Science Techniques; Bracco, G.; Holst, B., Eds.; Springer Series in Surface Science 2013, 51, 277-306; Springer-Verlag, Berlin, Heidelberg, New York, Tokyo.

(54) Moulder, J. F.; Stickle, W. E.; Sobol, P. E.; Bomben, K. D. Handbook of X-ray Photoelectron Spectroscopy, Chastian, J., Ed.; Perkin-Elmer Corp.: Eden Prairie, MN, 1992.

(55) Thome, J.; Himmelhaus, M.; Zharnikov, M.; Grunze, M. Increased Lateral Density in Alkanethiolate Films on Gold by Mercury Adsorption. Langmuir 1998, 14, 7435-7449.

(56) Ratner, M.; Castner, D. Electron Spectroscopy for Chemical Analysis, in Surface Analysis - The principal techniques; Vickerman, J. Ed.; Wiley: Chichester, 1997.

(57) Lamont, C. L. A.; Wilkes, J. Attenuation Length of Electrons in Self-Assembled Monolayers of $n$-Alkanethiols on Gold. Langmuir 1999, 15, 2037-2042.

(58) Leung, T. Y. B.; Schwartz, P.; Scoles, G.; Schreiber, F.; Ulman, A. Structure and Growth of 4-Methyl-4'-Merkaptobiphenyl Monolayers on Au(111): A Surface Diffraction Study. Surf. Sci. 2000, 458, 34-52. 
(59) Azzam, W.; Fuxen, C.; Birkner, A.; Rong, H.-T.; Buck, M.; Wöll, C. Coexistence of Different Structural Phases in Thioaromatic Monolayers on Au(111). Langmuir 2003, 19, 49584968.

(60) Schreiber, F. Self-Assembled Monolayers: From 'Simple' Model Systems to Biofunctionalized Interfaces. J. Phys.: Condens. Matter 2004, 16, R881-R900.

(61) Stöhr, J. NEXAFS Spectroscopy; Springer series in surface sciences ; 25; 1. ed., corr. 2. print.; Springer: Berlin [u.a.], 2003.

(62) Batson, P. E. Carbon-1s Near-Edge-Absorption Fine-Structure in Graphite. Phys. Rev. $B$ 1993, 48, 2608-2610.

(63) Ford, W. E.; Gao, D.; Knorr, N.; Wirtz, R.; Scholz, F.; Karipidou, Z.; Ogasawara, K.; Rosselli, S.; Rodin, V.; Nelles, G.; et al. Organic Dipole Layers for Ultralow Work Function Electrodes. ACS Nano 2014, 8, 9173-9180.

(64) Querebillo, C.; Terfort, A.; Allara, D.; Zharnikov, M. Static Conductance of NitrileSubstituted Oligophenylene and Oligo(phenylene ethynylene) Self-Assembled Monolayers Studied by Mercury-Drop Method. J. Phys. Chem. C 2013, 117, 25556-25561.

(65) Chiechi, R. C.; Weiss, E. A.; Dickey, M. D.; Whitesides, G. M. Eutectic GalliumIndium (EGaIn): A Moldable Liquid Metal for Electrical Characterization of Self-Assembled Monolayers. Angew. Chem. Int. Ed. 2008, 120, 148-150.

(66) Blum, V.; Gehrke, R.; Hanke, F.; Havu, P.; Havu, V.; Ren, X.; Reuter, K.; Scheffler, M. Ab Initio Molecular Simulations with Numeric Atom-Centered Orbitals. Comput. Phys. Commun. 2009, 180, $2175-2196$.

(67) Perdew, J. P.; Burke, K.; Ernzerhof, M. Generalized Gradient Approximation Made Simple. Phys. Rev. Lett. 1996, 629, 453 - 462.

(68) Ruiz, V. G.; Liu, W.; Zojer, E.; Scheffler, M.; Tkatchenko, A. Density-Functional Theory with Screened van der Walls Interactions for the Modeling of Hybrid InorganicOrganic Systems. Phys. Rev. Lett. 2012, 108, 146103

(69) Tkatchenko, A.; Scheffler, M. Accurate Molecular van der Waals Interactions from Ground-State Electron Density and Free-Atom Reference Data. Phys. Rev. Lett. 2009, 102, 073005 .

(70) Taucher, T. C.; Hehn, I.; Hofmann, O. T.; Zharnikov, M.; Zojer, E. Understanding Chemical versus Electrostatic Shifts in X-Ray Photoelectron Spectra of Organic SelfAssembled Monolayers. J. Phys. Chem. C 2016, 120, 3428-3437.

(71) Hughes, G.; Wang, C.; Batsanov, A. S.; Fearn, M. J.; Frank, S.; Bryce, M. R.; Perepichka, I. F.; Monkman, A. P.; Lyons, B. P. New Pyrimidine- and Fluorene-Containing 
Oligo(arylene)s: Synthesis, Crystal Structures, Optoelectronic Properties and a Theoretical Study. Org. Biomol. Chem. 2003, 1, 3069-3077.

(72) Ballav, N.; Schüpbach, B.; Dethloff, O.; Feulner, P.; Terfort, A.; Zharnikov, M. Direct Probing Molecular Twist and Tilt in Aromatic Self-Assembled Monolayers. J. Am. Chem. Soc. 2007, 129, 15416-15417.

(73) Kreis, M.; Bräse, S. A General and Efficient Method for the Synthesis of SilylProtected Arenethiols from Aryl Halides or Triflates, Adv. Synth. Catal. 2005, 347, 313-319.

(74) Barone, V.; Commisso, L.; Lelj, F.; Russo, N. Conformational Behaviour of Phenylpyrimidines. A Quantum Mechanical Study. Tetrahedron 1985, 41, 1915-1918.

(75) Kondoh, H.; Iwasaki, M.; Shimada, T.; Amemiya, K.; Yokoyama, T.; Ohta, T.; Shimomura, M.; Kono, S. Adsorption of Thiolates to Singly Coordinated Sites on Au(111) Evidenced by Photoelectron Diffraction. Phys. Rev. Lett. 2003, 90, 066102-1.

(76) Roper, M. G.; Skegg, M. P.; Fisher, C. J.; Lee, J. J.; Dhanak, V. R.; Woodruff, D. P.; Jones, R. G. Atop Adsorption Site of Sulphur Head Groups in Gold-Thiolate Self-Assembled Monolayers. Chem. Phys. Lett. 2004, 389, 87-91.

(77) Lüssem, B.; Müller-Meskamp, L.; Karthäuser, S.; Homberger, M.; Simon, U.; Waser, R. Electrical and Structural Characterization of Biphenylethanethiol SAMs. J. Phys. Chem. C 2007, 111, 6392-6397.

(78) Shaporenko, A.; Cyganik, P.; Buck, M.; Terfort, A.; Zharnikov, M. Self-Assembled Monolayers of Aromatic Selenolates on Noble Metal Substrates. J. Phys. Chem. B 2005, 109, 13630-13638.

(79) Charbonneau, G.-P.; Delugeard, Y. Structural Transition in Polyphenels. III. Crystal Structure of Biphenyl at 110 K. Acta Crystallogr. 1976, B32, 1420-1423.

(80) Matei, D. G.; Muzik, H.; Gölzhäuser, A.; Turchanin, A. Structural Investigation of 1,1'-Biphenyl-4-thiol Self-Assembled Monolayers on Au(111) by Scanning Tunneling Microscopy and Low-Energy Electron Diffraction. Langmuir 2012, 28, 13905-13911.

(81) Heister, K.; Johansson, L. S. O.; Grunze, M.; Zharnikov, M. A Detailed Analysis of the C 1s Photoemission of N-Alkanethiolate Films on Noble Metal Substrates. Surf. Sci. 2003, $529,36-46$.

(82) Cabellos, J. L.; Mowbray, D. J.; Goiri, E.; El-Sayed, A.; Floreano, L.; de Oteyza, D. G.; Rogero, C.; Ortega, J. E.; Rubio, A. Understanding Charge Transfer in Donor-Acceptor/Metal Systems: A Combined Theoretical and Experimental Study. J. Phys. Chem. C 2012, 116, 17991-18001. 
(83) El-Sayed, A.; Borghetti, P.; Goiri, E.; Rogero, C.; Floreano, L.; Lovat, G.; Mowbray, D. J.; Cabellos, J. L.; Wakayama, Y.; Rubio, A.; et al. Understanding Energy-Level Alignment in Donor - Acceptor/Metal Interfaces from Core-Level Shifts. ACS Nano 2013, 7, 6914-6920.

(84) Greenler, R. G. Infrared Study of Adsorbed Molecules on Metal Surfaces by Reflection Techniques. J. Chem. Phys. 1966, 44, 310-315.

(85) Parikh, A. N.; Allara, D. L. Quantitative Determination of Molecular Structure in Multilayered Thin Films of Biaxial and Lower Symmetry from Photon Spectroscopies. I. Reflection Infrared Vibrational Spectroscopy. J. Chem. Phys. 1992, 96, 927-945.

(86) Horsley, J.; Stöhr, J.; Hitchcock, A. P.; Newbury, D. C.; Johnson, A. L.; Sette, F. Resonances in the $K$ Shell Excitation Spectra of Benzene and Pyridine: Gas Phase, Solid, and Chemisorbed States. J. Chem. Phys. 1985, 83, 6099-6107.

(87) Yokoyama, T.; Seki, K.; Morisada, I.; Edamatsu, K.; Ohta, T. X-Ray Absorption Spectra of Poly-p-Phenylenes and Polyacenes: Localization of $\pi^{*}$ Orbitals. Phys. Scr. 1990, $41,189-192$.

(88) Bolognesi, P.; O’Keeffe, P.; Ovcharenko, Y.; Coreno, M.; Avaldi, L.; Feyer, V.; Plekan, O.; Prince, K. C.; Zhang, W.; Carravetta, V. Pyrimidine and Halogenated Pyrimidines Near Edge X-ray Absorption Fine Structure Spectra at C and N K-Edges: Experiment and Theory. $J$. Chem. Phys. 2010, 133, 034302.

(89) Lin, Yi-S.; Lin, H.-Ru; Liu, W.-L.; Lee, Y. T.; Tseng, C.-M.; Ni, C.-K.; Liu, C.-L.; Tsai, C.-C.; Chen, J.-L.; Hu, W.-P. Measurement and Prediction of the NEXAFS Spectra of Pyrimidine Andpurine and the Dissociation Following the Core Excitation. Chem. Phys. Lett. 2015, 636, 146-153.

(90) Zharnikov, M.; Frey, S.; Heister, K.; Grunze, M. An Extension of the Mean Free Path Approach to X-ray Absorption Spectroscopy. J. Electron Spectr. Relat. Phenom. 2002, 124, 15 24.

(91) Zharnikov, M.; Kuller, A.; Shaporenko, A.; Schmidt, E.; Eck, W. Aromatic SelfAssembled Monolayers on Hydrogenated Silicon. Langmuir 2003, 19, 4682-4687.

(92) Verwüster, E.; Wruss, E.; Zojer, E.; Hofmann, O. T. Exploring the Driving Forces behind the Structural Assembly of Biphenylthiolates on Au(111). J. Chem. Phys. 2017, 147, 024706.

(93) Vackář, J.; Hyt'ha, M.; Šimůnek, A. All-Electron Pseudopotentials. Phys. Rev. B 1998, 58, 12712-12720. 
(94) Methfessel, M.; Fiorentini, V.; Oppo, S. Connection between Charge Transfer and Alloying Core Level Shifts Based on Density-Functional Calculations. Phys. Rev. B 2000, 61, $5229-5236$.

(95) Morikawa, Y. ; Hayashi, T. ; Liew, C. C. ; Nozoye, H. First-Principles Theoretical Study of Alkylthiolate Adsoprtion on Au(111). Surf. Sci. 2002, 507, 46-50.

(96) Bagus, P. S.; Ilton, E. S.; Nelin, C. J. The Interpretation of XPS Spectra: Insights into Materials Properties. Surf. Sci. Rep. 2013, 68, 273-304.

(97) Shaporenko, A.; Terfort, A.; Grunze, M.; Zharnikov, M. A detailed analysis of the photoemission spectra of basic thioaromatic monolayers on noble metal substrates. J. Electr. Spec. Rel. Phenom. 2006, 151, 45-51.

(98) Bolognesi, P.; O’Keeffe, P.; Feyer, V.; Plekan, O.; Prince, K.; Coreno, M.; Mattioli, G.; Amore Bonapasta, A.; Zhang, W.; Carravetta, V.; et al. Inner Shell Excitation, Ionization and Fragmentation of Pyrimidine. J. Phys.: Conf. Ser. 2010, 212, 012002.

(99) Natan, A.; Kronik, L.; Haick, H.; Tung, R. T. Electrostatic Properties of Ideal and Non-Ideal Polar Organic Monolayers: Implications for Electronic Devices. Adv. Mater. 2007, $19,4103-4117$.

(100) Heimel, G.; Rissner, F.; Zojer, E. Modeling the Electronic Properties of $\pi$ Coniugated Self-Assembled Monoayers. Adv. Mater. 2010, 22, 2494-2513.

(101) Monti, O. L. A. Understanding Interfacial Electronic Structure and Charge Transfer: An Electrostatic Perspective. J. Phys. Chem. Lett. 2012, 3, 2342-2351.

(102) Rissner, F.; Egger, D. A.; Romaner, L.; Heimel, G.; Zojer, E. The Electronic Structure of Mixed Self-Assembled Monolayers. ACS Nano 2010, 4, 6735-6746.

(103) Chen, C.-Y.; Wu, K.-Y.; Chao, Y.-C.; Zan, H.-W.; Meng, H.-F.; Tao, Y.-T. Concomitant Tuning of Metal Work Function and Wetting Property with Mixed SelfAssembled Monolayers. Org. Electron. 2011, 12, 148-153.

(104) Vetushka, A.; Bernard, L.; Guseva, O.; Bastl, Z.; Plocek, J.; Tomandl, I.; Fejfar, A.; Baše, T.; Schmutz, P. Adsorption of Oriented Carborane Dipoles on a Silver Surface: Adsorption of Oriented Carborane Dipoles on a Silver Surface. Phys. Stat. Sol. B 2016, 253, 591-600.

(105) Kovalchuk, A.; Abu-Husein, T.; Fracasso, D.; Egger, D. A.; Zojer, E.; Zharnikov, M.; Terfort, A.; Chiechi, R. C. Transition Voltages Respond to Synthetic Reorientation of Embedded Dipoles in Self-Assembled Monolayers. Chem. Sci. 2016, 7, 781-787.

(106) Xiao, X.; Xu, B.; Tao, N. J. Measurement of Single Molecule Conductance:

Benzenedithiol and Benzenedimethanethiol. Nano Lett. 2004, 4, 267-271. 
(107) Danilov, A.; Kubatkin, S.; Kafanov, S.; Hedegård, P.; Stuhr-Hansen, N.; MothPoulsen, K.; Bjørnholm, T. Electronic Transport in Single Molecule Junctions: Control of the Molecule-Electrode Coupling through Intramolecular Tunneling Barriers. Nano Lett. 2008, 8, $1-5$.

(108) Obersteiner, V.; Egger, D. A.; Zojer, E. Impact of Anchoring Groups on Ballistic Transport: Single Molecule vs Monolayer Junctions. J. Phys. Chem. C 2015, 119, 21198-21208.

(109) C. Van Dyck, V. Geskin, J. Cornil, Fermi Level Pinning and Orbital Polarization Effects in Molecular Junctions: The Role of Metal Induced Gap States. Adv. Funct. Mater. 2014, 24, 6154-6165.

(110) Kovalchuk, A.; Egger, D. A.; Abu-Husein, T.; Zojer, E.; Terfort, A.; Chiechi, R. C. Dipole-Induced Asymmetric Conduction in Tunneling Junctions Comprising Self-Assembled Monolayers. RSC Adv. 2016, 6, 69479-69483.

(111) Yoon, H. J.; Bowers, C. M.; Baghbanzadeh, M.; Whitesides, G. M. The Rate of Charge Tunneling Is Insensitive to Polar Terminal Groups in Self-Assembled Monolayers in $\mathrm{Ag}^{\mathrm{TS}} \mathrm{S}\left(\mathrm{CH}_{2}\right)_{\mathrm{n}} \mathrm{M}\left(\mathrm{CH}_{2}\right)_{\mathrm{m}} \mathrm{T} / / \mathrm{Ga}_{2} \mathrm{O}_{3} / \mathrm{EGaIn}$ Junctions. J. Am. Chem. Soc. 2014, 136, 16-19.

(112) Zhang, G.-P.; Hu, G.-C.; Song, Y.; Li, Z.-L.; Wang, C.-K. Modulation of Rectification in Diblock Co-oligomer Diodes by Adjusting Anchoring Groups for Both Symmetric and Asymmetric Electrodes. J. Phys. Chem. C 2012, 116, 22009-22014.

(113) Trotter, J. The Crystal and Molecular Structure of Biphenyl. Acta Crystallogr. 1961, $14,1135-1140$. 


\section{TOC Graphic}

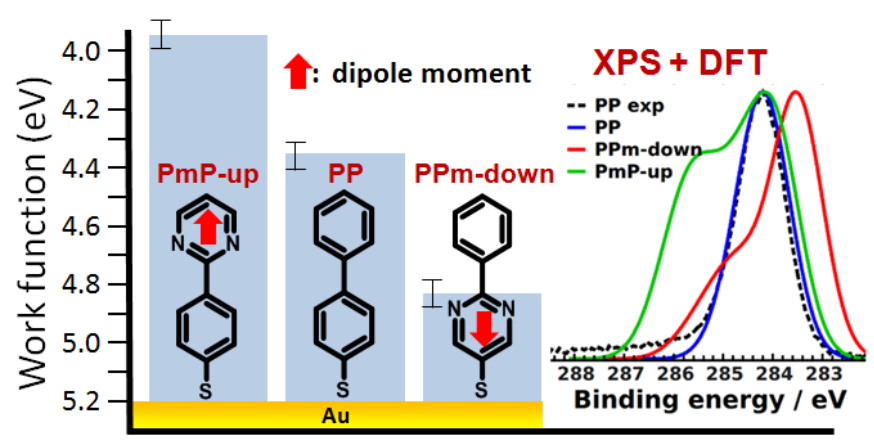

